\title{
The Majorana Demonstrator Neutrinoless Double-Beta Decay Experiment
}

\author{
N. Abgrall, ${ }^{1}$ E. Aguayo, ${ }^{2}$ F. T. Avignone III, ${ }^{3,4}$ A. S. Barabash, ${ }^{5}$ F. E. Bertrand, ${ }^{4}$ M. Boswell, ${ }^{6}$ \\ V. Brudanin, ${ }^{7}$ M. Busch, ${ }^{8,9}$ A. S. Caldwell, ${ }^{10}$ Y.-D. Chan, ${ }^{1}$ C. D. Christofferson, ${ }^{10}$ \\ D. C. Combs, ${ }^{8,11}$ J. A. Detwiler, ${ }^{12}$ P. J. Doe, ${ }^{12}$ Yu. Efremenko, ${ }^{13}$ V. Egorov, ${ }^{7}$ H. Ejiri, ${ }^{14}$ \\ S. R. Elliott, ${ }^{6}$ J. Esterline, ${ }^{8,9}$ J. E. Fast, ${ }^{2}$ P. Finnerty, ${ }^{8,15}$ F. M. Fraenkle, ${ }^{8,15}$ \\ A. Galindo-Uribarri, ${ }^{4}$ G. K. Giovanetti, ${ }^{8,15}$ J. Goett, ${ }^{6}$ M. P. Green, ${ }^{8,15}$ J. Gruszko, ${ }^{12}$ \\ V. E. Guiseppe, ${ }^{16}$ K. Gusev, ${ }^{7}$ A. L. Hallin, ${ }^{17}$ R. Hazama, ${ }^{14}$ A. Hegai, ${ }^{1}$ R. Henning, ${ }^{8,15}$ \\ E. W. Hoppe, ${ }^{2}$ S. Howard, ${ }^{10}$ M. A. Howe, ${ }^{8,15}$ K. J. Keeter, ${ }^{18}$ M. F. Kidd, ${ }^{19}$ A. Knecht, ${ }^{12}$ \\ O. Kochetov, ${ }^{7}$ S. I. Konovalov, ${ }^{5}$ R. T. Kouzes, ${ }^{2}$ B. D. LaFerriere, ${ }^{2}$ J. Leon, ${ }^{12}$ \\ L. E. Leviner, ${ }^{8,11}$ J. C. Loach, ${ }^{1,20}$ P. N. Luke, ${ }^{21}$ S. MacMullin, ${ }^{8,15}$ R. D. Martin, ${ }^{1}$ S. Mertens, ${ }^{1}$ \\ L. Mizouni, ${ }^{2,3}$ M. Nomachi, ${ }^{14}$ J. L. Orrell, ${ }^{2}$ C. O'Shaughnessy, ${ }^{8,15}$ N. R. Overman, ${ }^{2}$ \\ David Phillips, ${ }^{8,11}$ A. W. P. Poon, ${ }^{1}$ K. Pushkin, ${ }^{16}$ D. C. Radford, ${ }^{4}$ K. Rielage, ${ }^{6}$ \\ R. G. H. Robertson, ${ }^{12}$ M. C. Ronquest, ${ }^{6}$ A. G. Schubert, ${ }^{12}$ B. Shanks, ${ }^{8,15}$ T. Shima, ${ }^{14}$ \\ M. Shirchenko, ${ }^{7}$ K. J. Snavely, ${ }^{8,15}$ N. Snyder, ${ }^{16}$ D. Steele, ${ }^{6}$ J. Strain, ${ }^{8,15}$ A. M. Suriano, ${ }^{10}$ \\ J. Thompson, ${ }^{17}$ V. Timkin, ${ }^{7}$ W. Tornow, ${ }^{8,9}$ R. L. Varner, ${ }^{4}$ S. Vasilyev, ${ }^{13}$ K. Vetter, ${ }^{1}$ \\ K. Vorren, ${ }^{8,15}$ B. R. White, ${ }^{4}$ J. F. Wilkerson, ${ }^{4,8,15}$ T. Williams, ${ }^{4}$ W. Xu, ${ }^{6}$ E. Yakushev, ${ }^{7}$
} A. R. Young, ${ }^{8,11}$ C.-H. Yu, ${ }^{4}$ and V. Yumatov ${ }^{5}$

\footnotetext{
${ }^{1}$ Nuclear Science Division, Lawrence Berkeley National Laboratory, Berkeley, CA 94720, USA

${ }^{2}$ Pacific Northwest National Laboratory, Richland, WA 99352, USA

${ }^{3}$ Department of Physics and Astronomy, University of South Carolina, Columbia, SC 29208, USA

${ }^{4}$ Oak Ridge National Laboratory, Oak Ridge, TN 37831, USA

${ }^{5}$ Institute for Theoretical and Experimental Physics, Moscow 117218, Russia

${ }^{6}$ Los Alamos National Laboratory, Los Alamos, NM 87545, USA

${ }^{7}$ Joint Institute for Nuclear Research, Dubna 141980, Russia

${ }^{8}$ Triangle Universities Nuclear Laboratory, Durham, NC 27708, USA

${ }^{9}$ Department of Physics, Duke University, Durham, NC 27708, USA

${ }^{10}$ South Dakota School of Mines and Technology, Rapid City, SD 57701, USA

${ }^{11}$ Department of Physics, North Carolina State University, Raleigh, NC 27695, USA

${ }^{12}$ Center for Experimental Nuclear Physics and Astrophysics and Department of Physics, University of Washington, Seattle, WA 98195, USA

${ }^{13}$ Department of Physics and Astronomy, University of Tennessee, Knoxville, TN 37996, USA

${ }^{14}$ Research Center for Nuclear Physics and Department of Physics, Osaka University, Ibaraki, Osaka 567-0047, Japan

${ }^{15}$ Department of Physics and Astronomy, University of North Carolina, Chapel Hill, NC 27599, USA

${ }^{16}$ Department of Physics, University of South Dakota, Vermillion, SD 57069, USA

${ }^{17}$ Centre for Particle Physics, University of Alberta, Edmonton, AB, Canada T6G $2 G 7$

${ }^{18}$ Department of Physics, Black Hills State University, Spearfish, SD 57799, USA

${ }^{19}$ Tennessee Tech University, Cookeville, TN 38505, USA

${ }^{20}$ Shanghai Jiaotong University, Shanghai 200240, China

${ }^{21}$ Engineering Division, Lawrence Berkeley National Laboratory, Berkeley, CA 94720, USA
} 
Correspondence should be addressed to S. R. Elliott; elliotts@lanl.gov

Received 28 June 2013; Accepted 7 August 2013; Published 29 January 2014

Academic Editor: Vincenzo Flaminio

Copyright (c) 2014 N. Abgrall et al. This is an open access article distributed under the Creative Commons Attribution License, which permits unrestricted use, distribution, and reproduction in any medium, provided the original work is properly cited. The publication of this article was funded by $\mathrm{SCOAP}^{3}$.

The Majorana Demonstrator will search for the neutrinoless double-beta $(\beta \beta(0 \nu))$ decay of the isotope ${ }^{76} \mathrm{Ge}$ with a mixed array of enriched and natural germanium detectors. The observation of this rare decay would indicate that the neutrino is its own antiparticle, demonstrate that lepton number is not conserved, and provide information on the absolute mass scale of the neutrino. The Demonstrator is being assembled at the 4850-foot level of the Sanford Underground Research Facility in Lead, South Dakota. The array will be situated in a low-background environment and surrounded by passive and active shielding. Here we describe the science goals of the Demonstrator and the details of its design.

\section{Introduction}

1.1. Neutrinoless Double-Beta Decay. Despite being discovered well over a decade ago [1-3], the incorporation of neutrino mass and mixing into the standard model (SM) of particle physics remains elusive. A minimalistic Higgs coupling leaves the SM fine-tuned, with the neutrino masses lying some 6 orders-of-magnitude or more below that of the other SM leptons. Avoiding such unnaturalness requires new physics. One highly attractive option, afforded by the electric neutrality of the neutrino, is the addition of a lepton-numberviolating Majorana mass term [4]. Majorana neutrinos have the novel property that particle and antiparticle are distinguished only by chirality. A Majorana mass term provides a natural explanation for the lightness of the SM neutrino via the seesaw mechanism $[5,6]$. Majorana neutrinos also provide plausible scenarios for leptogenesis capable of accounting for the excess of matter over antimatter in the observable universe $[7,8]$.

Neutrinoless double-beta $(\beta \beta(0 \nu))$ decay searches represent the only viable experimental method for testing the Majorana nature of neutrinos [9]. The observation of this process would immediately imply that lepton number is violated and that neutrinos are Majorana particles [10]. The decay rate may be written as

$$
\left(T_{1 / 2}^{0 \nu}\right)^{-1}=G^{0 v}\left|M_{0 \nu}\right|^{2}\left(\frac{\left\langle m_{\beta \beta}\right\rangle}{m_{e}}\right)^{2},
$$

where $G^{0 v}$ is a phase space factor including the couplings, $M_{0 \nu}$ is a nuclear matrix element, $m_{e}$ is the electron mass, and $\left\langle m_{\beta \beta}\right\rangle$ is the effective Majorana neutrino mass. The latter is given by

$$
\left\langle m_{\beta \beta}\right\rangle=\left|\sum_{i=1}^{3} U_{e i}^{2} m_{i}\right|,
$$

where $U_{e i}$ specifies the admixture of neutrino mass eigenstate $i$ in the electron neutrino.

Until very recently, the most sensitive limits on $\beta \beta(0 \nu)$ decay came from $\mathrm{Ge}$ detectors enriched in ${ }^{76} \mathrm{Ge}$, namely, the Heidelberg-Moscow experiment [11] and the IGEX experiment [12-14]. Recent results from the EXO-200 experiment $[15,16]$ and from the KamLAND-Zen experiment $[17,18]$ have claimed stronger bounds on neutrino mass. It is difficult, however, to determine the best limit because of the uncertainties in the nuclear matrix elements. Previous-generation ${ }^{76} \mathrm{Ge}$ experiments also yielded a claim of the direct observation of $\beta \beta(0 \nu)$ decay by Klapdor-Kleingrothaus [19]. This claim has not been widely accepted by the neutrino community [2022]. While the EXO-200 and KamLAND-Zen results are in conflict with this claim, the recent results from GERDA [23$25]$ show that the observed peak is not an indication of $\beta \beta(0 \nu)$ decay in a ${ }^{76} \mathrm{Ge}$ experiment, which can compare its results with the Klapdor-Kleingrothaus claim without depending on the nuclear matrix elements. For recent comprehensive experimental and theoretical reviews, see [26-35].

A measurement of the $\beta \beta(0 \nu)$ decay rate would yield information on the absolute neutrino mass. Measurements of atmospheric, solar, and reactor neutrino oscillation [36] indicate a large parameter space for discovery of $\beta \beta(0 \nu)$ decay just beyond current experimental bounds below $\left\langle m_{\beta \beta}\right\rangle \sim$ $50 \mathrm{meV}$. Moreover, evidence from the SNO experiment [2] of a clear departure from nonmaximal mixing in solar neutrino oscillation implies a minimum effective Majorana neutrino mass of $\sim 15 \mathrm{meV}$ for the inverted hierarchy scenario. This target is within reach of next-generation $\beta \beta(0 \nu)$ searches. An experiment capable of observing this minimum rate would therefore definitively determine the Majorana or Dirac nature of the neutrino for inverted hierarchical neutrino masses.

Recent developments in germanium detector technology make a $\beta \beta(0 \nu)$-decay search feasible using ${ }^{76} \mathrm{Ge}$. In this paper, we describe the MAJORANA DEMONSTRATor as an experimental effort under construction in the Sanford Underground Research Facility (SURF) whose goal is to demonstrate the techniques required for a definitive next-generation $\beta \beta(0 \nu)$-decay experiment with enriched Ge detectors. The Demonstrator will also test the Klapdor-Kleingrothaus claim and will be sensitive to other non- $\beta \beta(0 \nu)$ physics signals in Ge. A complementary effort in Ge with similar sensitivity, the GERDA experiment [37], is presently operating 
in the Laboratori Nazionali del Gran Sasso (LNGS). The GERDA and MAJORANA collaborations intend to join in a proposal for the construction of a tonne-scale experiment. A nearly background-free tonne-scale ${ }^{76} \mathrm{Ge}$ experiment would be sensitive to effective Majorana neutrino masses below $~ 20$ $\mathrm{meV}$, potentially covering the parameter space corresponding to the inverted neutrino-mass hierarchy.

1.2. Non- $\beta \beta(0 \nu)$ Physics with the Majorana Demonstrator. The Ge-detector design used by Majorana has an energy threshold of $\sim 500 \mathrm{eV}$. This low threshold not only is critical for reducing $\beta \beta(0 \nu)$ background (Section 5), but also, in combination with low backgrounds, opens up new physics programs for the Majorana DEMONSTRATOR. Recent experiments [38-41] have shown the sensitivity of P-type, PointContact (P-PC) Ge detectors to light WIMP $\left(<10 \mathrm{GeV} / c^{2}\right)$ dark matter via direct detection. A very recent excess of low-energy events reported by the CDMS collaboration [42] lends further motivation for doing such a measurement. The DEMONSTRATOR may improve the current light WIMP limits by two orders of magnitude [43].

In addition to light WIMPS, MAJORANA will also be sensitive to solar axions that interact in the Ge crystals via several possible axion-electron coupling mechanisms. One of these mechanisms of particular interest to MAJORANA relies on the Primakoff conversion of axions into photons within the Ge crystal lattice when a Bragg condition is satisfied $[44,45]$. This technique requires knowledge of the crystal axis orientation relative to the sun at all times to maximize sensitivity. The collaboration will measure the detector crystal orientation for this purpose. The Majorana Demonstrator can also search for solar axions generated by the bremsstrahlung mechanism in the sun [46] and detected by the axioelectric effect [47]. Since this axion spectrum peaks at about $0.6 \mathrm{keV}$ and falls sharply by an order of magnitude by about $3 \mathrm{keV}$, the low threshold and background are keys for this measurement.

Majorana will also be sensitive to Pauli exclusion principle violating (PEPV) decays [48]. In this process, an atomic electron in a Ge atom spontaneously transitions from an upper shell to the K-shell, resulting in 3-ground shell electrons. During this deexcitation, a photon of energy close to that of a K-shell X-ray $(10 \mathrm{keV})$ is emitted. The slight difference in energy is due to the extrascreening of the nucleus by the two K-shell electrons. The detection of this photon would indicate a PEPV decay. Given the large number of atoms present in $40 \mathrm{~kg}$ of Ge, this will be a sensitive test of PEPV effects.

P-PC detectors were originally proposed for detecting coherent nuclear scattering of reactor neutrinos [49] and there is interest in using HPGe detectors to do a similar measurement with higher energy neutrinos at the spallation neutron source at Oak Ridge National Laboratory or similar sources $[50,51]$. A cryostat full of natural germanium detectors, similar to that planned for the Demonstrator, deployed at a shallow underground site near the spallation neutron source target should have sufficient sensitivity to make an observation of this process. Such an effort could demonstrate the feasibility of P-PC technology for reactor monitoring and nuclear treaty verification.

\section{The Majorana Demonstrator: An Overview}

The Majorana Demonstrator is an array of enriched and natural germanium detectors that will search for the $\beta \beta(0 \nu)$ decay of the isotope ${ }^{76} \mathrm{Ge}$. The specific goals of the MAJORANA DEMONSTRATOR are

(1) demonstrate a path forward to achieve a background rate at or below $1 \mathrm{cnt} /(\mathrm{ROI}-\mathrm{t}-\mathrm{y})$ in the $4 \mathrm{keV}$ region of interest (ROI) around the $2039 \mathrm{keV} \mathrm{Q}$-value for ${ }^{76} \mathrm{Ge} \beta \beta(0 \nu)$ decay. This is required for tonnescale germanium-based searches that will probe the inverted hierarchy parameter space for $\beta \beta(0 \nu)$ decay;

(2) show technical and engineering scalability toward a tonne-scale instrument;

(3) test the Klapdor-Kleingrothaus claim [19];

(4) perform searches for physics beyond the standard model, such as the search for dark matter and axions.

MAJORANA utilizes the demonstrated benefits of enriched high-purity germanium (HPGe) detectors. These include intrinsically low-background source material, understood enrichment chemistry, excellent energy resolution, and sophisticated event reconstruction. The main technical challenge is the reduction of environmental ionizing radiation backgrounds by about a factor 100 below what has been achieved in previous experiments.

We have designed a modular instrument composed of two cryostats built from ultrapure electroformed copper, with each cryostat capable of housing over $20 \mathrm{~kg}$ of P-PC detectors. $\mathrm{P}-\mathrm{PC}$ detectors were chosen after extensive R\&D by the collaboration and each has a mass of about $0.6-1.0 \mathrm{~kg}$. The baseline plan calls for $30 \mathrm{~kg}$ of the detectors to be built from Ge material enriched to $86 \%$ in isotope 76 and $10 \mathrm{~kg}$ fabricated from natural $\mathrm{Ge}\left(7.8 \%{ }^{76} \mathrm{Ge}\right)$. The modular approach will allow us to assemble and optimize each cryostat independently, providing a fast deployment with minimum interference on already-operational detectors.

Starting from the innermost cavity, the cryostats will be surrounded by an inner layer of electroformed copper, an outer layer of oxygen-free high thermal conductivity (OFHC) copper, high-purity lead, an active muon veto, polyethylene, and borated polyethylene. The cryostats, copper, and lead shielding will all be enclosed in a radon exclusion box. The entire experiment will be located in a clean room at the $4850^{\prime}$ level (1478 m) of the Sanford Underground Research Facility (SURF) in Lead, South Dakota.

\section{The P-PC-Detector Technology}

At the heart of Majorana is its enriched p-type pointcontact HPGe detectors $[49,52]$. These detectors have all the benefits of coax HPGE detectors traditionally used for $\beta \beta(0 \nu)$, but also possess superb pulse-shape analysis (PSA) discrimination between single-site interactions (such as $\beta \beta(0 v)$-decay events) and multisite interaction events (such as Compton scattering of $\gamma$-ray backgrounds), making them 
highly suitable for $\beta \beta(0 \nu)$ searches. Their small capacitance results in superb energy resolution and a low-energy threshold, making them suitable for event correlation techniques using X-rays. Furthermore, they are relatively robust and simple to produce. Their simplicity has the advantage of reducing the characterization studies and detector-to-detector tuning required for an effective PSA algorithm.

Like coaxial Ge detectors, P-PC detectors are cylindrical in shape. The electron holes, however, are collected on a small, shallow contact, rather than a long-extended electrode as in a coaxial detector. In the detectors, made for MAJORANA, this "point contact" varies in diameter from about 2 to $6.5 \mathrm{~mm}$ and in depth from less than a micron (for implanted contacts) to a few $\mathrm{mm}$. Since they have no long inner contact, P-PC detectors are generally limited in their length-to-diameter aspect ratio. If a crystal is too long, it can result in having a "pinchoff" island of undepleted material in the center, especially if the Point-Contact end of the crystal has a lower net impurity concentration than the opposite end. This can be alleviated by using crystals with larger impurity gradients and ensuring that the point contact is placed at the end of the crystal where the material is of higher impurity (generally the seed end).

The Majorana collaboration has procured $20 \mathrm{~kg}$ of natural-germanium modified-BEGe detectors from CANBERRA industries [53], modified so as not to have the thin front window that permits sensitivity to low-energy external $\gamma$-rays. These detectors typically have masses in the range of 600-700 $\mathrm{g}$ and use a thin, implanted contact. Detectors from enriched ${ }^{76} \mathrm{Ge}$ material are being produced by AMETEK/ ORTEC [54]. These detectors have a mass of around $1 \mathrm{~kg}$ each, with a greater length-to-diameter ratio than that of the BEGe detectors. We anticipate that approximately $30 \mathrm{~kg}$ of these detectors will be produced for the Majorana DEMONSTRATOR from the $41.6 \mathrm{~kg}$ of $86 \%$ enriched ${ }^{76} \mathrm{Ge}$ material supplied to AMETEK/ORTEC.

Figure 1 illustrates our modeling of a sample P-PC detector, $5 \mathrm{~cm}$ in diameter and $5 \mathrm{~cm}$ long. The color scale shows hole drift speeds, in $\mathrm{mm} / \mathrm{ns}$, the black lines show charge drift trajectories, and the light grey lines show "isochrones"-loci of equal hole drift time for events in the detector bulk. We have adapted such drift-time calculations to create a PSA heuristic for GEANT4 simulations (see Section 5.3) of the remaining background in the Majorana Demonstrator following the PSA cut. For this PSA heuristic, multiple interactions within an event are examined for their relative drift times, and that information is combined with each individual energy deposit to determine whether the PSA algorithm would be capable of rejecting the event.

Measured signals from a P-PC detector are shown in Figure 2. Both current and charge pulses are shown, for both a single-site (a) and a multisite (b) $\gamma$-ray events. The difference in signal shape is readily apparent, with four distinct interactions evident in (b). The MAJORANA collaboration uses two different types of PSA algorithm to discriminate between these two classes of events. The first of these, developed by the GERDA collaboration [55], compares the maximum height of the current pulse $(A)$ to the total energy of the event $(E)$ as determined from the height of the charge pulse. Multiple

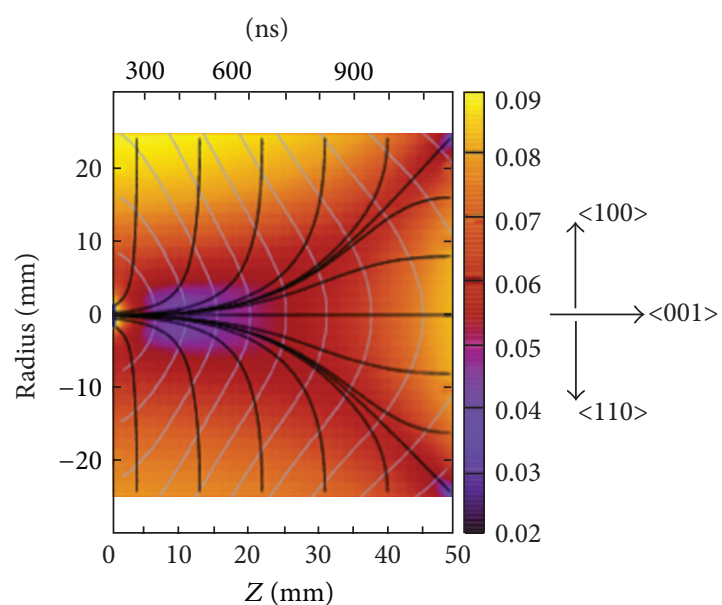

FIGURE 1: Calculated hole drift in a $5 \mathrm{~cm}$ diameter $\times 5 \mathrm{~cm}$ long P-PC detector.

interactions result in multiple charge pulses separated in time and therefore in a reduced value of $A / E$.

An alternative approach [56] uses a library of unique, measured single-site signals to perform event-by-event $\chi^{2}$ fitting of experimental pulse shapes. A method for building this library from a large number of measured signals has been developed and tested with simulation and experimental studies. Results of this optimized PSA algorithm on P-PC data are shown in Figure 3, where the high spectrum is for all events from a ${ }^{232} \mathrm{Th}$ source, and the low spectrum is for events that pass the PSA cut. The strong peak remaining is the double-escape peak from the $2615 \mathrm{keV}^{208} \mathrm{Tl} \gamma$-ray, which is a proxy for single-site $\beta \beta(0 \nu)$-decay events. The algorithm retains at least $95 \%$ of these events while rejecting up to $99 \%$ of the single-escape, multisite events. One should compare this to the A/E results of [55] where the double-escape peak events are accepted at $89 \%$ and the single-escape peaks are rejected at $93 \%$.

More recently, we have also developed a model for slow, partial-energy events from interactions within the lithium contact layer on the outer edge of P-PC detectors. This layer is typically $1 \mathrm{~mm}$ in thickness but is not entirely inactive material. Hence, events within that layer can produce signals with long rise-times and partial-energy collection. We now understand these signals as the result of competition between the diffusion of holes out of the Li layer with the recombination of electrons and holes at Li precipitates $[57,58]$. Since these slow events can potentially generate much of the background at very low energies, a detailed and comprehensive understanding of this process is crucial for dark matter, axion, and other low energy-dominated physics sensitivity.

\section{The Majorana Demonstrator Construction and Facility}

4.1. Enrichment, Ge Reduction and Refinement, and Detector Production. The Demonstrator baseline plan calls for $30 \mathrm{~kg}$ of enriched Ge detectors. The Collaboration acquired $42.5 \mathrm{~kg}$ 

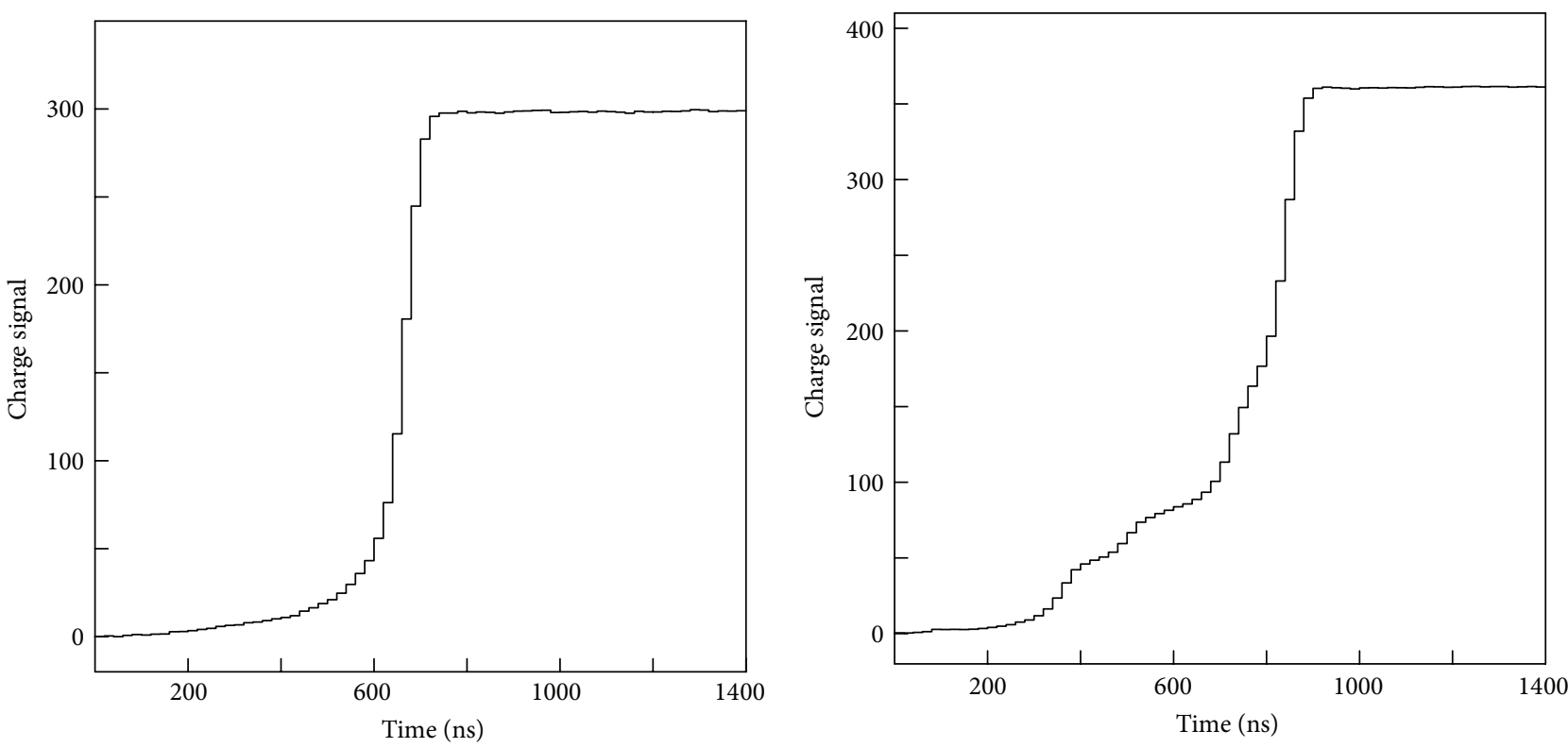

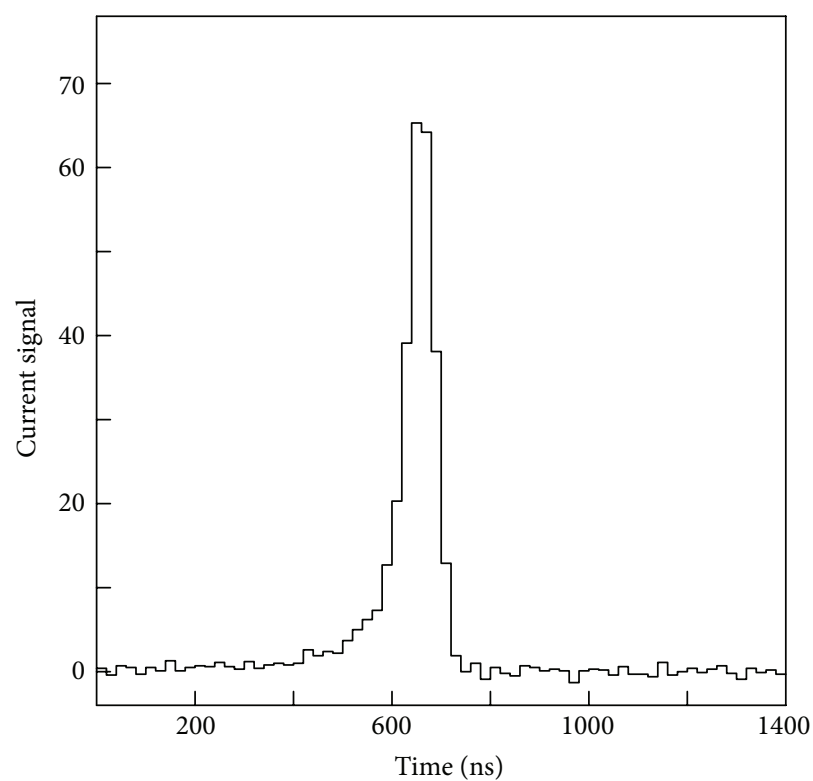

(a)

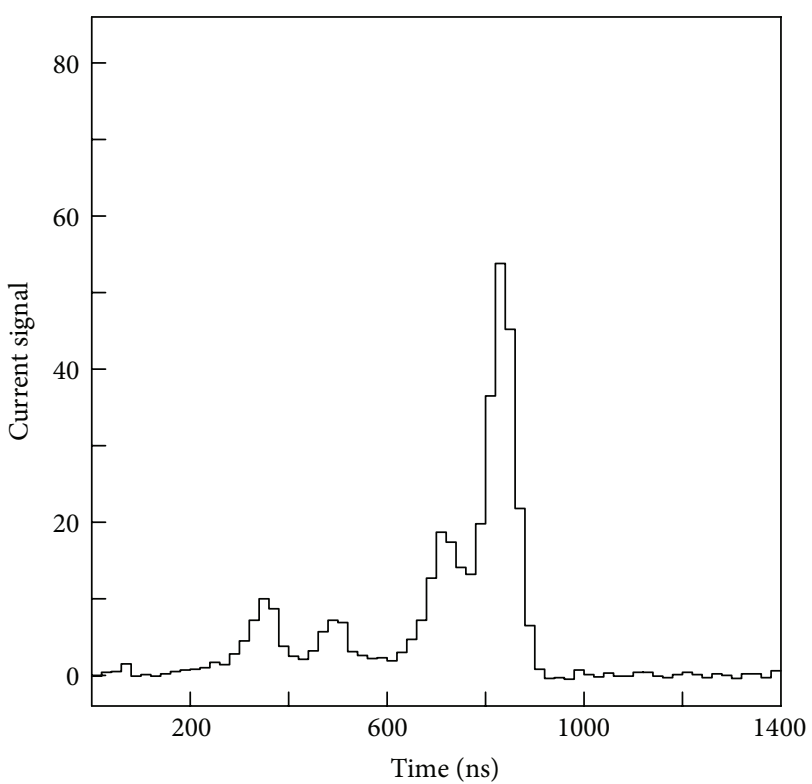

(b)

FIGURE 2: Current and charge pulse responses of a P-PC detector to single- and multisite $\gamma$-ray events. The pulse shapes in (a) show the charge (top) and current (bottom) signals resulting from a typical single-site interaction, while (b) shows how the pulse-shape response to a multisite interaction is clearly different.

of ${ }^{76} \mathrm{Ge}$ in the form of $60.5 \mathrm{~kg}_{\text {of }}{ }^{76} \mathrm{GeO}_{2}$, which was produced by the Joint Stock Company Production Association Electrochemical Plant (ECP) in Russia. The order was delivered to Oak Ridge, TN, United States, in two shipments. The first $20 \mathrm{~kg}$ was delivered in September 2011, while the rest was delivered in October 2012. A special steel container was constructed to minimize the exposure of the enriched ${ }^{76} \mathrm{Ge}$ to cosmic rays during transport. The calculated cosmic ray production of ${ }^{68} \mathrm{Ge}$ and ${ }^{60} \mathrm{Co}$ is reduced by a factor 10 and 15 , respectively, for samples transported within this container. Shielded storage for the enriched material being processed in Oak Ridge is provided by a cave located about $8 \mathrm{~km}$ from the processing and detector manufacturing facilities. The cave has an overburden of $40 \mathrm{~m}$ of rock, which is more than adequate for shielding the enriched material from the hadronic component of cosmic rays.

Electrochemical Systems Inc. (ESI), in Oak Ridge, TN, provided the first stage of material preparation. Before processing any enriched material, pilot tests with natural $\mathrm{GeO}_{2}$ were performed to qualify the procedures. The delivered ${ }^{76} \mathrm{GeO}_{2}$ from ECP underwent a high-temperature reduction in a hydrogen atmosphere. When the resistivity of 


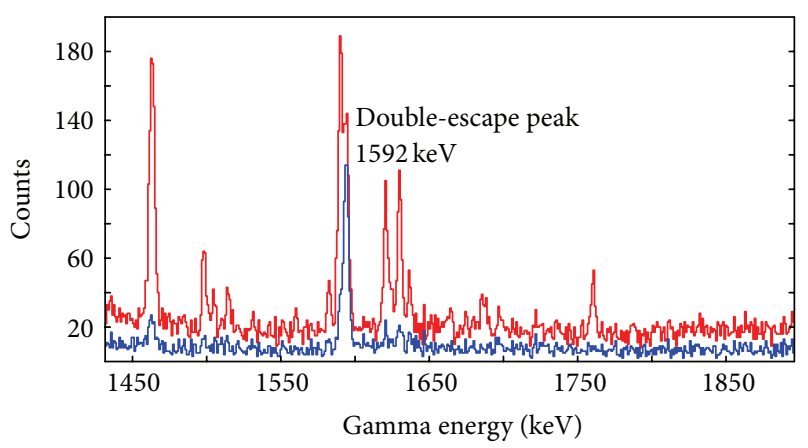

FIgURe 3: Pulse-shape analysis results for P-PC data. The red spectrum is for all events within the energy range, while the blue spectrum is for events that pass the PSA cut.

the reduced material was greater than $3 \Omega \mathrm{cm}$, the material was then purified by zone refinement to reach a resistivity of $>47 \Omega \mathrm{cm}$. The processing by ESI provided a yield of $98 \%$ of enriched material suitable for further refinement and detector manufacturing. In addition to the process qualification of the refinement at ESI, AMETEK/ORTEC produced two P-PC detectors fabricated from natural Ge that had been reduced and purified by ESI.

The refined enriched material from ESI was further purified by zone refining at AMETEK/ORTEC before being used as charge in a Czochralski crystal puller. Detector blanks were cut from the pulled crystals $(\sim 70 \mathrm{~mm}$ diameter), followed by the standard detector manufacturing steps of lithiation, implantation of the $\mathrm{p}^{+}$contact, and passivation. At each step, the mass of the enriched materials being handled was recorded for inventory control. In the production of the enriched P-PC detectors, slurries from detector cutting and shaping processes, as well as small samples cut from the pulled crystal for evaluation, were saved and reprocessed by ESI for reuse in detector production. Acids that were used in the detector manufacturing process were not saved. At all stages of the detector fabrication process, any enriched materials that were not being worked on were transported back to the cave for storage.

Once an enriched detector is manufactured, it is mounted in a PopTop capsule and tested for performance. The PopTop detector capsule, which can be detached from the cold finger, offers superior portability and ease of disassembly-qualities that are essential for transferring the detector from the capsule to the low-background string mounts used in the DEMONSTRATOR. In order to mitigate contamination of the mounted detector in a PopTop capsule, the parts that are in direct contact with the detector are made from only radiopure materials. For example, indium contacts are replaced by gold or clean tin contacts, and synthetic charcoal with low radon emanation rate is used to maintain the vacuum in the capsule.

The production of enriched detectors began in November 2012. As of April 2013, ten enriched detectors with a total mass of approximately $9.5 \mathrm{~kg}$ have been fabricated with eight delivered to SURF. The detectors were transported by ground to SURF, and a portable muon counter [59] was used to log the cosmic-ray exposure during the trip.
4.2. Detector Array Configuration. The detector array for Demonstrator is designed with many goals in mind. The functional requirements are as follows.

(i) Only the most radiopure materials are used to construct the detector holder. All the detector and string components are made out of two possible materials: underground electroformed $\mathrm{Cu}(\mathrm{UGEFCu})$ from the temporary clean room (TCR) (Section 4.3.2) or NXT85 (a Teflon that is specially manufactured in a clean room environment, Section 5). The UGEFCu has a maximum thickness of $1.27 \mathrm{~cm}$ and the NXT- 85 parts are fabricated from $15.875 \mathrm{~cm}$ long rods that are $3.175 \mathrm{~cm}$ in diameter. The NXT-85 mass is minimized and used only where electrical insulation is required.

(ii) UGEFCu and NXT-85 parts are processed in the cleanroom machine shop (Section 4.7), so designs must be compatible with the machine tools purchased for this shop. Use of wire electric discharge machining $(\mathrm{EDM})$ is preferred as a clean material removal technique.

(iii) A $5 \mathrm{~mm}$ vacuum gap or $1 \mathrm{~mm}$ of NXT-85 is required to isolate high voltage from neutral components.

(iv) The detectors have variable dimensions in order to maximize the yield of enriched germanium. Unique parts are minimized in order to allow a wide range of detector sizes to be packaged while still providing a high packing factor of germanium in each cryostat.

(v) Threaded connections are difficult to produce and keep clean. We minimized the number of threaded connections, and where that was not practical, special methods are employed to ensure quality, cleanliness, and repeatability of threaded connections.

Each detector is housed in a frame referred to as a detector unit (Figure 4). The crystal mounting plate (CMP) is the foundation, while 3 hollow hex rods and high-voltage (HV) nuts provide connection to the HV ring, which clamps the detector in place. The crystal insulators provide electrical isolation between the HV surface of the detector and the neutral CMP. The crystal insulators are also sized to compensate for the different coefficients of thermal expansion of copper and germanium, providing equivalent clamping force when operating warm and cold.

The crystal insulators snap into place on the CMP, as does the contact pin bushing. The contact pin and low mass front end (LMFE) board are held in place by the spring clip, which provides contact pressure between the pin and detector. The spring clip is held in place and tensioned by parylene-coated number $4-40 \mathrm{Cu}$ nuts on thread-milled studs in the CMP. Parylene coating of these nuts provides a higher strength connection than that of NXT-85 nuts while providing thread lubrication to prevent copper galling.

Up to five detector units are stacked into a string (Figure 5). The string is clamped together with tie rods. The string adapter plate connects the string to the coldplate. The tie rod bottom nuts and adapter plate nuts, also parylene coated, provide a strong clamping force for thermal contact. 


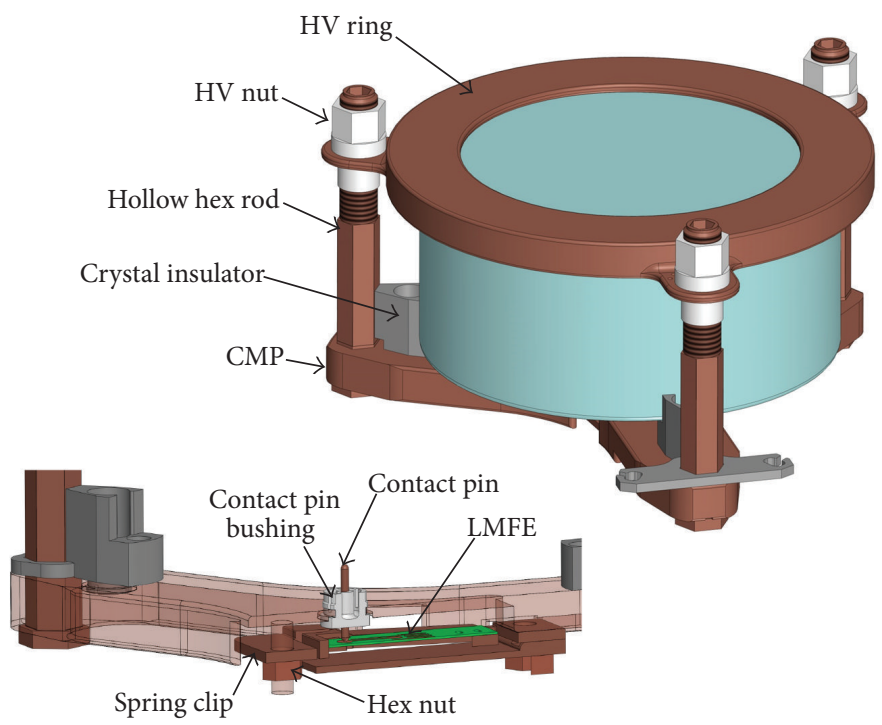

Figure 4: A rendering of the detector unit design (see text for details).

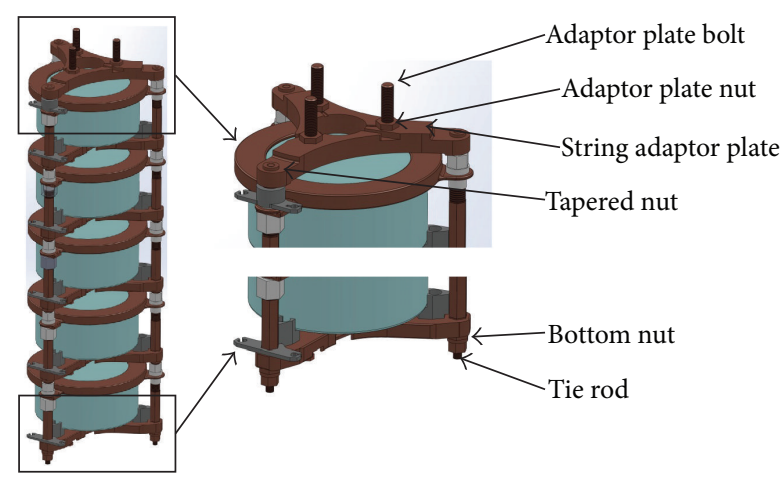

FIgURE 5: A rendering of the string design (see text for details).

The LMFE board is built on a $0.025 \mathrm{~cm}$ thick fused silica substrate, with all of the cold electronics mounted on the board. The LMFE assembly is then inserted into spring fingers on the spring clip. This assembly is then mounted to the CMP. After the contact pin and detector are mounted, the spring clip tensioning nut is set to apply the appropriate contact pressure.

The detector units are designed to accommodate a wide range of detector sizes from multiple vendors. Detectors have the shape of a right cylinder and can have a diameter of 50-77 $\mathrm{mm}$ and a height up to $65 \mathrm{~mm}$. Variations in diameter and corner sharpness can be accounted for by producing custom crystal insulators. Variations in the details of the pin contact with respect to the overall shape are accounted for by having 2 different pin lengths. Variations of less than $0.5 \mathrm{~mm}$ in this geometry can be accounted for by adjusting the spring clip tension starting point.

The special methods for threaded connections include the use of dedicated tools, as with all parts machined underground, to avoid cross contamination. The number 440 studs on the CMP are thread milled from bulk material, so instead of having independent screws, there are in place studs that protrude from the interior surface. Interior threads are made using roll-form taps, which produce a consistent thread quality and no burr. All interior threads are of the smallest depth necessary for strength, and there are no blind tapped holes. This makes cleaning and drying parts easier. All threaded parts are verified by hand before release for final cleaning.

\subsection{The Electroformed Copper Cryostats, the Thermosyphon, and the Vacuum System}

4.3.1. The Majorana Demonstrator Module. The MajorANA DEMONSTRATOR is a modular instrument as the detector strings are deployed in two copper cryostats, each is outfitted with its own vacuum and cryogenic systems for independent operation. This modular scheme allows for phased deployment of detectors as they become available and suggests a scheme for development of a tonne-scale

${ }^{76} \mathrm{Ge}$ experiment; a larger experiment can be constructed by deploying several similar cryogenic modules. The cryostats designed for the DEMONSTRATOR are each capable of housing seven of the previously described detector strings, for a total capacity of $\sim 20 \mathrm{~kg}$ of HPGe detectors apiece. The first cryostat, Cryostat 1, will contain detectors produced from both natural and enriched germanium. The second cryostat, Cryostat 2, will only contain detectors produced from enriched germanium. The cryostats are constructed from copper: a design decision motived by the ability to produce ultrapure copper through chemical electroforming. Cryostats 1 and 2 are fabricated from this ultrapure copper, while an initial prototype cryostat is fabricated from commercially sourced copper. The prototype cryostat will only contain 
two strings of detectors produced from natural germanium. It serves as a testbed for mechanical designs, fabrication methods, and assembly procedures that will be used for the construction of the electroformed copper Cryostats 1 and 2.

4.3.2. Electroforming. The primary requirement for the copper used in the Majorana Demonstrator is that it is sufficiently purified. This includes removal of naturally occurring radioactivity from $U$ and $T h$, as well as the elimination and prevention of reformation of cosmogenic radioisotopes species. Due to its large total mass, the radiopurity goal for the copper that is used in the inner shield and detector components is very stringent. To attain the background goal of $3 \mathrm{cnts} /(\mathrm{ROI}-\mathrm{t}-\mathrm{y})$, the required purity levels are $<0.3 \mu \mathrm{Bq}^{238} \mathrm{U} /$ $\mathrm{kg} \mathrm{Cu}$ (or $2.4 \times 10^{-14} \mathrm{~g}^{238} \mathrm{U} / \mathrm{g} \mathrm{Cu}$ ), and $<0.3 \mu \mathrm{Bq}^{232} \mathrm{Th} / \mathrm{kg} \mathrm{Cu}$ (or $7.5 \times 10^{-14} \mathrm{~g}^{232} \mathrm{Th} / \mathrm{g} \mathrm{Cu}$ ). Electroforming copper in a carefully controlled manner within a clean environment allows one to produce copper with the required radiopurity [60].

A secondary requirement for the electroformed copper relates to its physical properties. The mechanical properties of electroformed copper can vary drastically depending on the conditions under which it was formed. Conditions that favor high purity can form large crystalline structures with poor mechanical strength. Small polycrystalline formations can exhibit adequate tensile strength but lower purities. These conditions, which are seemingly at odds with one another, require that a careful balance of operational parameters should be obtained in the electroforming production process.

Design considerations for load bearing components were carried out using conservative estimates for material properties such as yield strength. The design yield stress value used for electroformed copper was estimated to be $48 \mathrm{MPa}$. Mechanical testing and evaluation is necessary to prove the plated material's ability to withstand the loading conditions without failure. Mechanical evaluation has shown the yield strength to be $83.2 \mathrm{MPa}$ [61] on average with a significant degree of strain hardening observed. The UGEFCu has, therefore, shown compliance with the design criteria.

The electroformed material for the DEMonstrator has been fabricated mostly from cylinders that are up to $35.6 \mathrm{~cm}$ in diameter (the inside diameter of the cryostats). The thermosyphon was formed on a mandrel $1.90 \mathrm{~cm}$ in diameter. The copper produced can range from a few tens of microns to very thick plates near $1.4 \mathrm{~cm}$. Time constraints are the primary limitation when producing very thick electroforms. The current plating rate for the DEMONSTRATOR's copper is typically from 38 to $64 \mu \mathrm{m}$ per day, depending on a variety of parameters. While this rate can be increased, it is at the expense of purity and mechanical properties of the electrodeposited material. From the plating rate indicated, a $1.4 \mathrm{~cm}$ thick electroform takes approximately 8-12 months to complete.

4.3.3. Cryostat Design. The cryostat is a copper vacuum enclosure that includes an electron-beam-welded vessel assembly along with removable top and bottom lids (see Figure 6). The vacuum seals between these components are a custom MAJORANA design that uses thin ( $51 \mu \mathrm{m}$ thick) parylene gaskets sandwiched between tapered surfaces machined into the copper components. Copper rail sectors and clamp bolts are used to maintain parallelism during assembly and pump down. Vacuum forces are sufficient to maintain the seal, so bolt strength is not a factor in effective sealing.

Detector strings are mounted to a copper coldplate, which rests on Vespel pins that provide support and alignment while maintaining a thermal break from the room temperature vacuum vessel. An infrared (IR) shield is mounted to the underside of the coldplate to reduce detector leakage current generated by IR radiation. The cryostat is supported at its crossarm by a copper frame (not shown in Figure 6) inside the lead stack. A transition is made to stainless steel conflat vacuum hardware at the far end of the crossarm tube via a copper/stainless explosion-bonded transition flange. All of the stainless steel vacuum hardware is located outside of the Demonstrator's passive shielding.

4.3.4. Vacuum System. Each cryostat is mounted to its own vacuum system and constructed from all-metal ultrahigh vacuum components. A $200 \mathrm{lpm}$ oil-free diaphragm pump provides rough vacuum, a 300 lps turbo-molecular pump is used for initial pump down to UHV pressures, and a 1500 lps cryopump is used for steady state operation. A nonevaporable getter (NEG) pump is used to remove built-up noncondensable gasses. A residual gas analyzer provides mass spectrometry analysis of the vacuum. All of the active components, including valves, are remotely operable, and an application has been developed to monitor and control the system, allowing full-remote operation (see Section 4.5). Pressures are continuously uploaded to a slow-control database for history viewing.

4.3.5. Cryogenics. The detector strings are cooled via the coldplate by means of a thermosyphon [62]. The thermosyphon is a closed tube within the crossarm that joins the coldplate to a condenser volume residing outside the shield and inside a dewar containing liquid nitrogen. The thermosyphon contains nitrogen that is liquefied at the condenser and transported by gravity down the length of the crossarm to the coldplate, where, as it evaporates, it cools the coldplate. The evaporated nitrogen travels back to the condenser where it is reliquefied. In this cycle, heat is transported from the coldplate to liquid nitrogen in the dewar. The liquid nitrogen in the dewar then evaporates and is replenished from an external supply. The dual-phase nitrogen within the thermosyphon has a large effective thermal conductivity providing the required cooling power for the DEMONSTRATOR. The operating temperature can be tuned by adjusting the amount of thermosyphon nitrogen. By producing only a thin layer of condensed nitrogen at the coldplate, microphonics from evaporation is minimized.

The thermosyphon system consists of a thermosyphon tube, custom liquid nitrogen dewar, gaseous $\mathrm{N}_{2}$ plumbing for loading nitrogen into the tube, and an external ballast tank. The thermosyphon tube is constructed from the same 

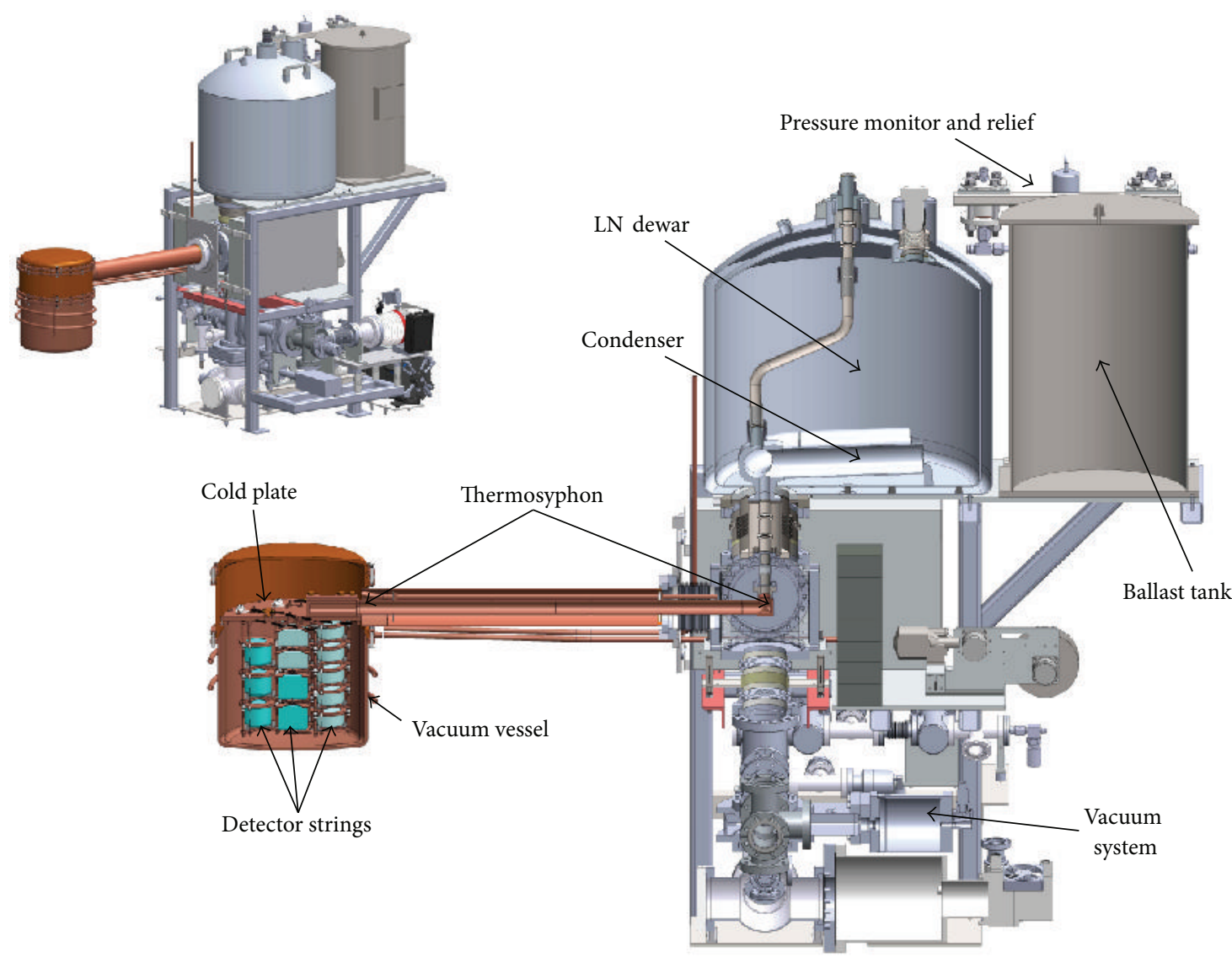

Figure 6: The Majorana Demonstrator module. Detector Strings are housed within ultralow background cryostats, each of which are supplied with its own vacuum and cryogenic systems (see text for details of vacuum and cryogenic system function).

grade of UGEFCu as that from which the cryostat is fabricated. The liquid nitrogen dewar is a custom-fabricated device which includes the condenser volume with an in-vacuum connection to the thermosyphon tube and an in-air connection to the $\mathrm{N}_{2}$ supply. The external ballast tank is primarily a safety feature, allowing for evaporation of condensed thermosyphon-tube nitrogen in the case of a loss of liquid nitrogen supply, without creating a hazardous overpressure condition. Additionally, since the ballast tank is isolated from the thermosyphon tube, nitrogen can be stored for several ${ }^{222} \mathrm{Rn}$ half-lives (3.8 days) before it is loaded into the thermosyphon tube. In this way we can ensure that the nitrogen circulated in the thermosyphon tube is radon-free.

4.4. Detector Acceptance, Characterization, and Calibration. There are a number of experimental characteristics that need to be monitored during the course of the experiment including:

(i) energy scale and linearity;

(ii) absolute efficiency for double-beta decay within each detector;

(iii) energy resolution and peak shape; (iv) background and signal tagging efficiencies;

(v) pulse waveforms response.

We have developed a three-phase plan to ensure that the required data are acquired. The phases include acceptance, characterization, and calibration. The acceptance testing is the initial phase of evaluating the detector performance. This type of testing is done upon receipt of the detectors from their manufacturers and is performed in the transport cryostats with the original manufacturer-supplied preamplifiers. The tests conducted during this phase are relatively cursory and meant solely to establish that the received detectors meet some minimum qualifications. The basic tests performed are energy scale and resolution, relative efficiency, leakage current or capacitance at depletion, initial estimate of the dead layer, and detector mass and dimensions.

Characterization measurements are conducted to fully determine the operating behavior of a detector. This includes energy scale, resolution, capacitance measurements, singlesite and multiple-site event separation performance, deadlayer measurements, and crystal-axis measurements. Characterization measurements are done with detectors in the final string configuration both within a test cryostat and within 
the Demonstrator cryostat prior to operation, that is, prior to commissioning.

The calibration measurements are designed to monitor the stability of the system during run-time operation. Initially, hour-long calibrations with a ${ }^{228}$ Th source will be conducted on a weekly basis. These runs will measure the stability of the energy scale and resolution, efficiency, and pulse-shape analysis (PSA) efficiency. Once the stability of the detectors has been established, the time period between the source calibrations can be extended to twice monthly or even monthly.

In the detector and string characterization stage, measurements are being performed with button sources $\left({ }^{133} \mathrm{Ba}\right.$, ${ }^{60} \mathrm{Co}$, and ${ }^{241} \mathrm{Am}$ ) in a clean room environment. Once the detector strings are loaded into the cryostat, access will be limited. Each monolith will have a low-background source pathway of PTFE tubing that spirals around the outside of the cryostat. A line source of ${ }^{228}$ Th will be remotely fed into the pathway, enabling the calibration of the entire cryostat with a single source either during final testing or after the monolith is placed in the shield. Simulations have shown that in an hour-long run we can accumulate the necessary statistics to monitor the efficiency, PSA performance, energy scale, and resolution while simultaneously keeping the count rate below the signal pile-up threshold of $\sim 100 \mathrm{~Hz}$. The source will be parked in an external garage separated from the shield-penetrating section of the pathway by an automated valve system. During calibration runs, the valve will be open, and the entire pathway will be purged with liquid nitrogen boil-off. During production runs, the shield-penetrating section of the pathway will be sealed off from the garage. The source itself will be encased in two plastic tubes to prevent leaving residual radioactivity within the pathway.

\subsection{Electronics and Data Acquisition}

4.5.1. The Detector Readout Electronics. Each of the two cryostat modules in the DEMONSTRATOR contains seven strings, with each string holding up to five detectors. Figure 7 illustrates the basic design of the low-noise, low-radioactivity signal-readout electronics. It consists of the LMFE [63], a circuit containing the input FET and feedback components, which is located close to the detector in order to minimize stray input capacitance. It also includes the preamplifier, which lies outside the cryostat and is connected to the LMFE by a long cable. The main challenges to the practical realization of this design are sourcing components for the front end that are low in both noise and radioactivity and dealing with the long cable in the feedback loop.

The LMFE is a resistive feedback circuit. This architecture has various benefits over the common pulse reset alternative; namely, that it is simpler and avoids interference from reset pulses: a problem for multiple-detector systems. The LMFE is $20.5 \times 7 \mathrm{~mm}^{2}$ in size and weights approximately $80 \mathrm{mg}$. The substrate is fused silica, which was chosen for its high radiopurity, low dielectric losses, and low thermal conductivity. Its low thermal conductivity means that a significant temperature gradient can be maintained across the board. By

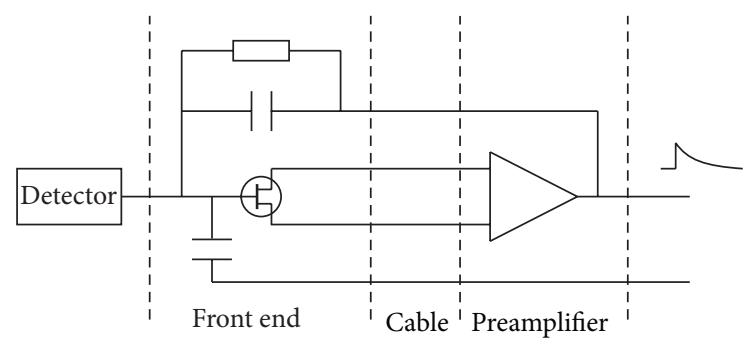

FIGURE 7: A high-level illustration of the signal readout scheme for each detector channel (see text for details on this readout design and the typical values for the components).

choosing an appropriate geometry and thermal conductivity for the substrate, the FET can be maintained at its optimal operating temperature for noise performance by self-heating which is adjustable by controlling drain to source voltage. The chosen FET is a bare Moxtek MX-11 JFET low-noise die with high transconductance and very low-input capacitance. It is attached to the silica board with silver epoxy via its gate substrate, while the source and drain pads are connected to traces with Al-Si wire bonds. The feedback resistor is formed by sputtering a layer of amorphous $\mathrm{Ge}(\mathrm{a}-\mathrm{Ge})$ and has a resistance of $\sim 10-100 \mathrm{G} \Omega$ at cryogenic temperatures. The feedback capacitance of $\sim 0.2 \mathrm{pF}$ is formed by the stray capacitance between traces on the front end.

Extraordinarily, low-noise levels can be achieved using the front end design previously described. The equivalent noise charge achieved without a detector was $55 \mathrm{eV}$ FWHM, and with a detector, a small P-PC, it was $85 \mathrm{eV}$. The noise levels are not representative of what will be achieved in the DEMONSTRATOR because of the greater detector capacitance, but they indicate that the noise resulting from the materials and components of the front end board will not be the limiting factor. To minimize the material budget and thermal dissipation in the cryostat, a $0.4 \mathrm{~mm}$ diameter, $\sim 2 \mathrm{~m}$ long miniature $50 \Omega$ coaxial cable is used to drive signals from the LMFE to the first stage of the preamplifier, based on a classicfolded cascode transistor design. The preamp. is capable of rise times below $10 \mathrm{~ns}$ with judicious choice of front end components and a short cable connecting it to the front end. With the long cable used in the DEMONSTRATOR configuration, this rise time increases to $\sim 40-70 \mathrm{~ns}$, depending on the length of the cable.

The preamplifiers are organized by string position on motherboards. On the motherboard, each detector has a lowgain and a high-gain signal output for digitization, resulting in a maximum of 70 channels per cryostat. The digitization electronics for each cryostat are in separate VME crates. Each crate has a single-board computer to read out the digitizers in that crate and the entire system is controlled by a central DAQ computer. A conceptual schematic of the data acquisition electronics is given in Figure 8.

The collaboration is using GRETINA digitizer boards [64] developed as part of the GRETINA experiment (we gratefully thank the GRETINA collaboration for digitizer board loans). The GRETINA cards are a combination of a 


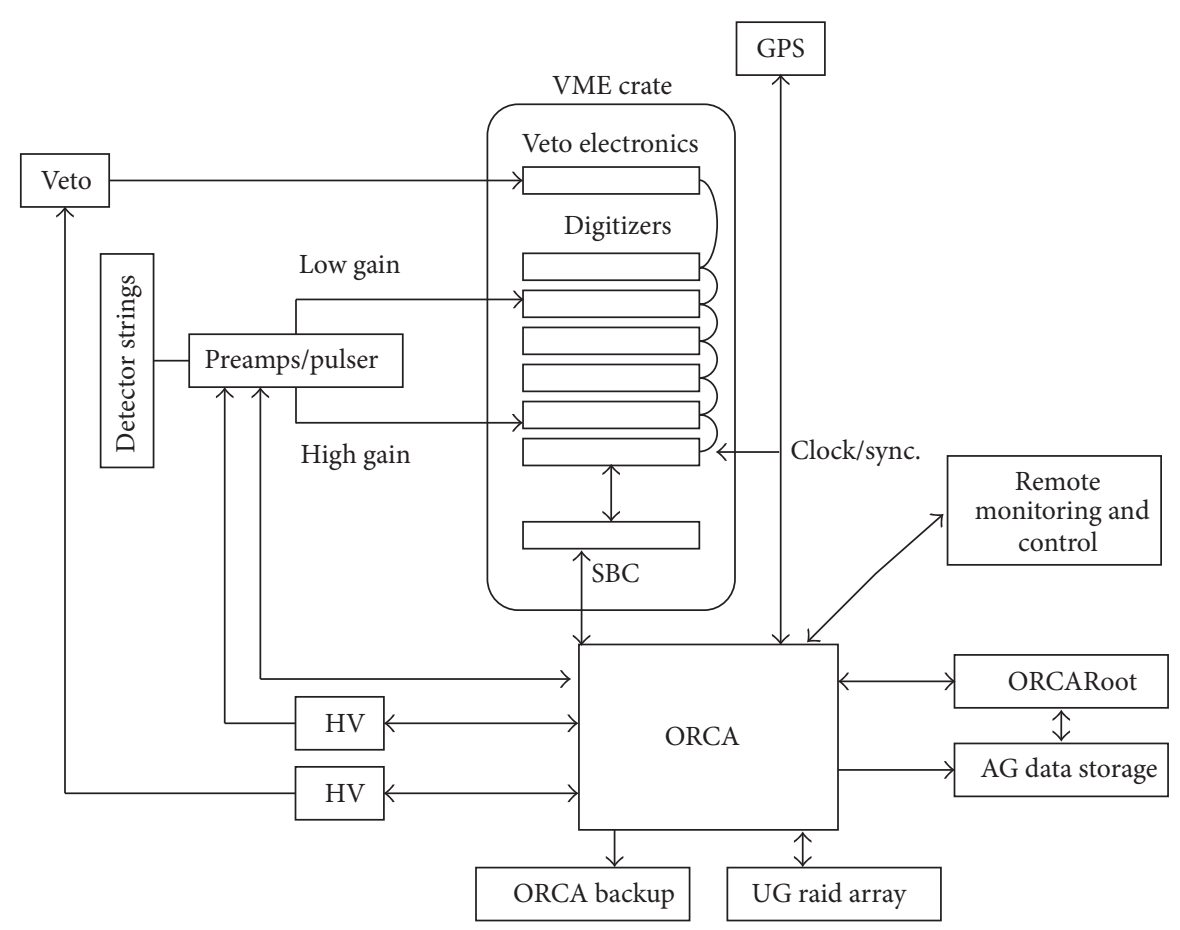

FIGURE 8: A high-level schematic of the DAQ system. Each detector uses a low- and a high-gain digitizer channel for a total of 70 channels per crate. Each VME crate has one single-board computer (SBC) that reads out all electronics in its crate, sending the resulting data to ORCA. Data is buffered locally underground and then transferred to the surface. There will be one VME crate per module. The veto electronics will reside only in one of the two crates.

digitizer and digital signal processor, which accept 10 inputs directly from the detector preamplifiers and digitize at a nominal frequency of $100 \mathrm{MHz}$ with 14 bit ADC precision. A field-programmable gate array (FPGA) performs digital leading edge and/or constant fraction discrimination, trapezoidal shaping, and pole-zero correction. Some special firmware extensions were specifically developed for the DemonstraTOR. These modifications allow for independent configurations of the 10 input channels that allow the traces to be decimated in such a way as to simultaneously capture a fully sampled rising edge with presummed regions before and after the edge for studying slow pulses. The capability of measuring the input trigger rate (triggers/second) for each individual input channel is another feature added to the card specifically for the Demonstrator. Data records are fixed at 2020 samples/event and the card allows these samples to be the sum of various ADC output values. This summing capability allows the card to achieve selectable time windows $(20,40$, 80,160 , and $200 \mu \mathrm{s}$ ) for the acquired data while maintaining a constant event length. In the case of the DEMONSTRATOR, different crystal geometries can be plugged into the same card, and therefore it is mandatory to be able to accommodate the different time constants of the different crystals.

A controller card communicates between the motherboard and the digitization electronics. The controller card contains 16 pulse generator outputs with individually controlled amplitudes, 16 DAC levels outputs for setting the drain-to-source voltage of the FETs, and 16 ADC inputs to monitor the first stage outputs of the preamplifiers. The pulser outputs of the controller card are used for electronics calibration, monitoring the gain stability of independent channels, and monitoring the trigger efficiency. The controller cards are implemented in the overall DAQ system and slow-control processor.

4.5.2. Other Data Acquisition and Electronic Systems. The passive lead shield is surrounded by scintillator panels that are used for an anticoincidence (veto) shield. The veto (see Section 4.6) has separate electronics in one of the VME crates with some additional electronics in a separate NIM BIN. Data from the veto is read out by the main DAQ software and integrated into the detector data stream. All veto events are time stamped using a scaler that counts the common clock pulses.

To ensure accurate time-stamping of the signals, a common clock from a global positioning system (GPS) module is distributed among the digitizer cards and the veto system. A common reset is used as a system synchronization pulse to simultaneously reset counters on all digitization and veto boards, thus providing an absolute time reference for each event.

Separate computer-controlled HV bias supply systems are used for the detector array and the photomultipliers of the veto shield. Each detector and phototube are powered by an independent $\mathrm{HV}$ channel, allowing for optimization of the 


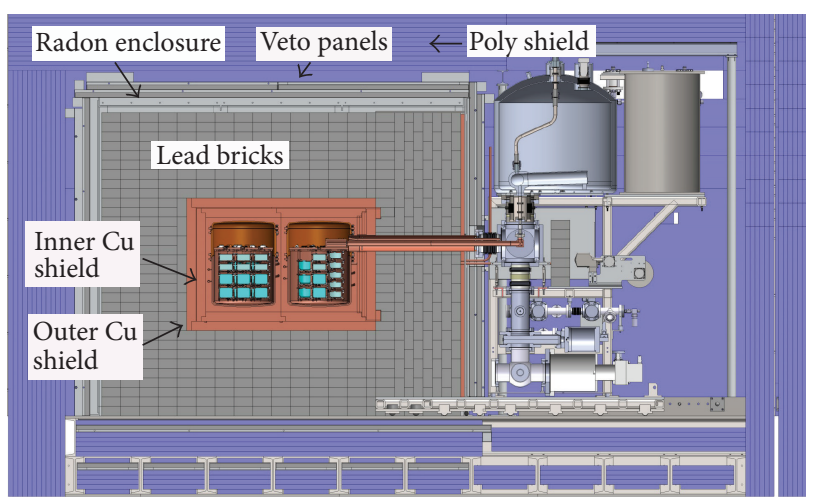

FIGURE 9: The shield system in cross section, shown with both cryostats installed.

HV setting for each detector and enabling any detector to be taken offline without affecting the rest of the array.

Data are transferred from the DAQ computer to an underground 32 TB RAID system and from there to the aboveground analysis computer system. The underground RAID storage is used as a buffer to prevent loss of data in the case of an underground-to-surface network failure.

4.5.3. The Data Acquisition Software. The DAQ software system used by the Demonstrator is the object-oriented realtime control and acquisition (ORCA) [65] application. ORCA is a general purpose, highly modular, object-oriented, acquisition, and control system that can be configured at run time to represent different hardware configurations and data readout schemes by dragging items from a catalog of objects into a configuration window. Since each object is composed of its own fully encapsulated data structures as well as support and diagnostic code, ORCA can easily support specific experiments, such as the Demonstrator.

4.6. The Shielding Configuration. The passive shield consists of graded shield materials starting outside of the cryostat and extending out to the overfloor table, or base plate, upon which the experiment is assembled. This system also includes the integral calibration source track and drive system, the transport mechanisms for installing and retracting the cryostat from the bulk of the shield for access, and a radon scrubbing and nitrogen delivery system for providing purge gas to the internal portions of the shield. A high-level summary of shield components is given in Table 1 , and the complete shield assembly is shown in Figure 9.

Gamma rays from the inner region of the shielding contribute to the background. Therefore, materials with extremely low radioactivity must be used in this region. Copper can be purchased very pure and made ultrapure via electroforming (see Section 4.3.2) to meet the specifications required by the background budget. The innermost layer of the shield will be $5 \mathrm{~cm}$ thick and built of UGEFCu sheet. The practical upper limit on the thickness for electroforming in our baths is about $1.40 \mathrm{~cm}$, so this shield is constructed of four layers of $1.25 \mathrm{~cm}$ thick plates.

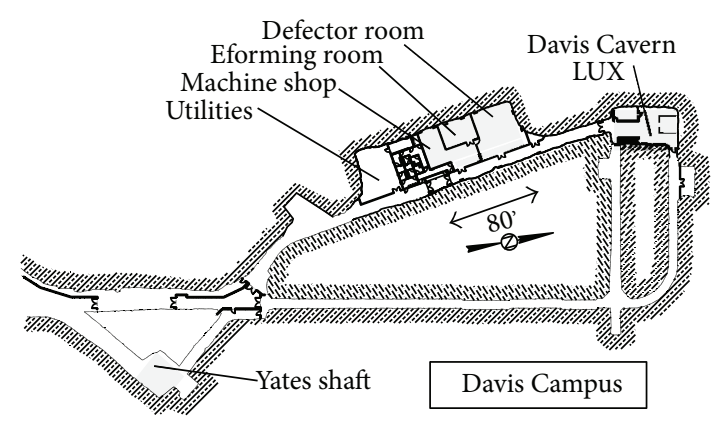

Figure 10: The layout of the Davis Campus at SURF showing the three Majorana labs and the location of the LUX experiment at the Davis Cavern. The corridor that joins the two projects is a clean space.

The outer copper shield is made of $5 \mathrm{~cm}$ thick OFHC copper plates and machined and bolted together to provide the mechanical reference points for the experiment. This shield "box" is supported off the overfloor table on four OFHC copper legs to allow for precision alignment and reference independent of the lead shield bricks. The lead shield consists of a $45 \mathrm{~cm}$ thick layer of $5.1 \times 10.2 \times 20.3 \mathrm{~cm}^{3}$ bricks to be stacked in place and machined as needed.

The copper and lead shield will be contained inside a semisealed aluminum box. This box will permit for the controlled purging of the gas within the inner cavity that contains the detector modules. The box is constructed of a welded and bolted aluminum structural frame, with $0.3175 \mathrm{~cm}$ thick bolted $\mathrm{Al}$ panels. All seams and openings are sealed, with the exception of the seals around the monolith, which are gasketed and bolted for easy removal and replacement.

Two layers of veto panels surround the radon exclusion box. Each panel consists of a $2.54 \mathrm{~cm}$ thick scintillating acrylic sheet. This sheet has small longitudinal grooves machined for wavelength-shifting fibers and is wrapped in a custom-made reflecting layer to compensate for light attenuation along the fibers. Light from the fibers is read out by a single $1.27 \mathrm{~cm}$ photomultiplier tube. The scintillator assembly is enclosed in a light-tight aluminum box. There are a total of 32 veto panels surrounding the radon exclusion box, including a number that reside within openings of the overfloor. Sheets of $2.54 \mathrm{~cm}$ thick high density polyethylene (HDPE) panels, stacked up to $30 \mathrm{~cm}$ of total thickness, make up the poly shield structure. The inner 2 layers consist of borated HDPE.

4.7. Underground Facilities. The Homestake Mine is home to the Sanford Underground Research Facility (SURF) that has been developed by the state of South Dakota as a site for experiments requiring underground laboratory space. The state of South Dakota, along with private donor T. Denny Sanford, has committed funds that have allowed refurbishment and access to underground space. Operation of SURF is funded by the U.S. Department of Energy and the state of South Dakota.

The MaJorana laboratory space at SURF consists of three cleanrooms in the Davis Campus complex (Figure 10), 
TABle 1: A summary of the shield components.

\begin{tabular}{lcr}
\hline Shield component & Material & Thickness \\
\hline Inner copper shield & 4 layers, $1.25 \mathrm{~cm}$ thick UGEFCu & $5 \mathrm{~cm}$ \\
Outer copper shield & OFHC copper, commercial & $5 \mathrm{~cm}$ \\
Lead shield & $5.1 \times 10.24 \times 20.3 \mathrm{~cm}^{3}$ bricks & $45 \mathrm{~cm}$ \\
Radon exclusion box & Al sheets & $0.32-0.635 \mathrm{~cm}$ \\
Veto panels & Scintillating acrylic & 2 layers, $2.54 \mathrm{~cm}$ each \\
Poly shield & HDPE & $30 \mathrm{~cm}$, inner layer borated \\
\hline
\end{tabular}

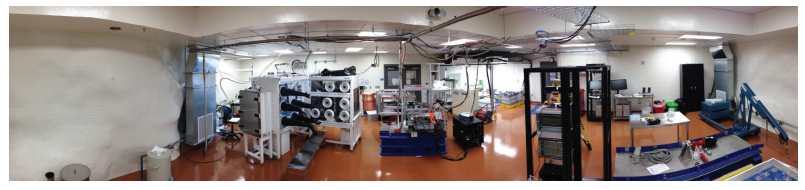

Figure 11: A view of the detector laboratory at the Davis Campus at SURF. In this view, the glove box is to the left, and the prototype module is at the center. In the rear, a soft-wall cleanroom is shown that serves the purpose of an area of increased cleanliness.

near the Yates shaft, at the $4850^{\prime}$ level ( $\sim 260$ m.w.e. [66]) of the mine. These rooms consist of a detector room, a general purpose lab space, and a machine shop. Figure 11 shows a picture of the detector room at SURF. This room will house the Demonstrator and has dimensions of $32^{\prime} \times 40^{\prime}$. Next to the detector room is the machine shop. Here, all the copper and NXT-85 parts for the Demonstrator are fabricated, thus further reducing the UGEFCu's surface exposure to cosmic rays. The machine shop is approximately $1000 \mathrm{ft}^{2}$ and includes two lathes, two mills, an oven, a wire electric discharge machine, a press, a drill press, and a laser engraver. The final room is a general purpose lab and is used for testing the detectors prior to their installation into the DemonstraTOR. The room is approximately $550 \mathrm{ft}^{2}$ and was originally designed for electroforming activities; hence, its formal name is the electroforming room.

The final Majorana laboratory is the temporary cleanroom (TCR) (not pictured in Figure 11), which sits on the same level as the Davis Campus and is approximately $1 \mathrm{~km}$ away. It consists of a cleanroom building, shown in Figure 12, that contains 10 electroforming baths and a small annex for changing into cleanroom garb. The total area of the building is $12^{\prime} \times 40^{\prime}$ with the annex room consuming $8^{\prime} \times 12^{\prime}$ of that area. The TCR was required, prior to beneficial occupancy of the Davis Campus, for initiating the slow process of electroforming copper in order for parts to be ready on time for assembly of the Demonstrator.

\section{The Background Model and the MajoranA Demonstrator Sensitivity}

The projected background in the Demonstrator is significantly improved over previous-generation experiments. This reduction is a result of fielding the detectors in large arrays

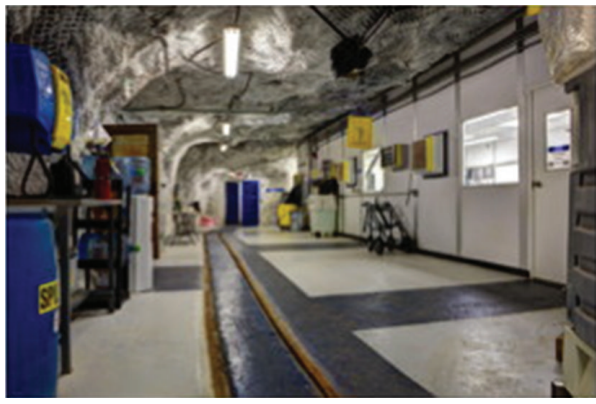

FIGURE 12: A photograph of the TCR used for electroforming on the $4850^{\prime}$ level at SURF.

that share a cryostat and minimizing the amount of interstitial material. Further background suppression is achieved through the aggressive reduction of radioactive impurities in construction materials and minimization of exposure to cosmic rays. Majorana will also make use of event signatures to reject backgrounds that do appear, including pulseshape characteristics, detector hit granularity, cosmic-ray veto tags, and single-site time correlations. In this section, we describe these aspects of the Majorana Demonstrator design and their impact on the projected backgrounds and physics sensitivity.

5.1. Pure Materials. The production process for enriched germanium detectors (enrichment, zone refining, and crystal growth) efficiently removes natural radioactive impurities from the bulk germanium. The cosmogenic activation isotopes, ${ }^{60} \mathrm{Co}$ and ${ }^{68} \mathrm{Ge}$, are produced in the crystals, while they are aboveground but can be sufficiently reduced by minimizing the time to deployment underground and by the use of passive shielding during transport and storage.

For the main structural material in the innermost region of the apparatus, we choose copper for its lack of naturally occurring radioactive isotopes and its excellent physical properties. By starting with the cleanest copper stock we have identified and then electroforming it underground to eliminate primordial radioactivity and cosmogenically produced ${ }^{60} \mathrm{Co}$, we have achieved several orders-of-magnitude background reduction over commercial alternatives. Electroformed copper will also be employed for the innermost passive, high Z-shield. Commercial copper stock is clean enough 
for use as the next layer of shielding. For all uses of copper, we have certified the cleanliness of samples via assay. Modern lead is available with sufficient purity for use as the bulk shielding material outside of the copper layers.

Several clean plastics are available for electrical and thermal insulation. For the detector supports, we use a pure polytetrafluoroethylene (PTFE), DuPont Teflon NXT-85. Thin layers of low-radioactivity parylene will be used as a coating on copper threads to prevent galling and for the cryostat seal. For the few weight-bearing plastic components requiring higher rigidity, we have sourced pure stocks of PEEK (polyether ether ketone), produced by Victrex, and Vespel, produced by DuPont.

The front-end electronics are also designed to be lowmass and ultralow background because they must be located in the interior of the array adjacent to the detectors in order to maintain signal fidelity. The circuit board is fabricated by sputtering thin traces of pure gold and titanium on a silica wafer, upon which a bare FET is mounted using silver epoxy. A G $\Omega$-level feedback resistance is provided by depositing intrinsically pure amorphous Ge. Detector contact is made via an electroformed copper pin with beads of low-background tin at either end. An electroformed copper spring provides the contact force. Our signal- and high-voltage cables are extremely low-mass miniature coaxial cable. We have worked with the vendor to cleanly fabricate the final product using pure stock that we provide for the conductor, insulation, and shield. Cable connectors within the cryostat are made from electroformed copper, PTFE, and the same silica circuit boards used for the front-end electronics.

The high material purities required for the MAJORANA DEMONSTRATOR necessitated the development of improved assay capabilities. These capabilities are needed not only to establish that the required purities can be achieved, but also to monitor construction processes to verify that cleanliness is maintained. We rely primarily on three assay methods: $\gamma$ ray counting, inductively coupled plasma mass spectrometry (ICP-MS), and neutron activation analysis (NAA).

5.2. Background Rejection. One key advantage of HPGe detectors is their inherent excellent energy resolution. Background rejection in our P-PC detectors is significant due to not only the energy resolution, but also array granularity (interdetector coincidences), pulse-shape discrimination, and event time correlation. These techniques rely on the differentiation of the spatial and temporal distributions of $\beta \beta(0 \nu)$-decay events from most background events. Background signals from radioactive decay often include a $\beta$ and/or one or more $\gamma$-rays. Such $\gamma$-rays frequently undergo multiple scatters over several centimeters. Since $\beta \beta(0 v)$ decay energy deposition typically occurs within a small volume $\left(\approx 1 \mathrm{~mm}^{3}\right)$, it is a single-site energy deposit. The two different topologies can be separated by PSA (see Section 3). Rejection of decay-product series using single-site timecorrelation analysis techniques is also possible in these ultralow event rate experiments. For example, ${ }^{68} \mathrm{Ga} \beta^{+}$decays can only result in background to $\beta \beta(0 \nu)$ decay if one of the annihilation $\gamma$-rays interacts in the same crystal that contains the $\beta^{+}$. Hence it is always a multiple-site energy deposit and we reject much of this background through PSA. In addition, however, ${ }^{68} \mathrm{Ga}$ decays are preceded by the electron capture decay of the parent ${ }^{68} \mathrm{Ge}$. The low threshold of the P-PC detectors permits additional rejection by a time-correlation cut with the ${ }^{68} \mathrm{Ge} 10 \mathrm{keV} \mathrm{K}$ and $1 \mathrm{keV} \mathrm{L}$ X-rays.

\subsection{Monte Carlo Simulations. The Majorana and GERDA} collaborations have jointly developed a simulation software framework, MAGE, that is based on the GEANT4 $[67,68]$ simulation toolkit. MAGE is used to simulate the response of ultralow radioactive background detectors for ionizing radiation. The development of a common simulation framework used by both collaborations reduces duplication of effort, eases comparison between simulated data and experiment, and simplifies the addition of new simulated detector geometries. The MAgE package is described in more detail in [69].

MAGE has interfaces with numerous external packages including software that simulates charge pulse generation in HPGe detectors. The MAGE framework contains the geometry models of common detector objects, experimental prototypes, test stands, and the Demonstrator itself. It also implements customized event generators, GEANT4 physics lists, and output formats. All of these features are available as class libraries that are typically compiled into a single executable. The user selects the particular experimental setup implementation at run time via macros. In the prototyping phase, the simulation was used as a virtual test stand guiding detector design, allowing an estimate of the effectiveness of proposed background reduction techniques, and providing an estimate of the experimental sensitivity. During operation, MAGE will be used to simulate and characterize backgrounds to determine the ultimate sensitivity of the experiment. It will also provide probability distribution functions (PDFs) for likelihood-based signal extraction analyses.

MaJORAnA has developed a detailed geometry of the Demonstrator in Mage that consists of more than 3, 800 components. The collaboration has performed detailed simulations of 60,000 background contributions from different isotopes in these components that required $60 \mathrm{kCPU}-\mathrm{hrs}$ on different Linux clusters. The simulations include estimates of the rejection efficiencies from the analysis cuts discussed in Section 5.2. The PSA cut efficiencies, in particular, are estimated using a heuristic calculation in which multiple interactions in a detector are examined for their relative drift time using isochrone maps such as that depicted in Figure 1. That information is combined with the energies of the interactions to determine whether the PSA algorithm would be capable of rejecting the event. The summary of the background expectation after all cuts is given in Figure 13. These simulation results were used for the current background estimates and will be used in future analyses of data.

The collaboration has also developed an automated validation suite that thoroughly tests all the critical physics processes that are being simulated by MAGE and GEANT4 against validated experimental data. This suite is run every time there is a major update to MAGE or GEANT4 to verify that the critical physics processes are not altered between versions. 


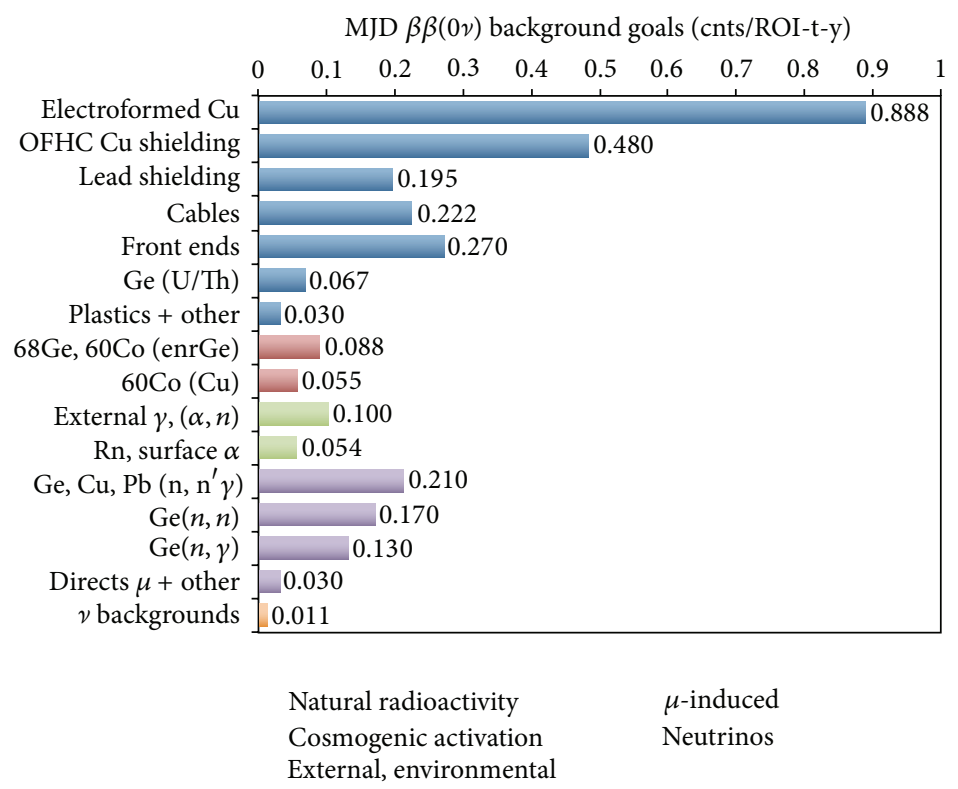

FIGURE 13: Estimated background contributions for the $\beta \beta(0 \nu)$-decay search. Backgrounds from natural radioactivity are shown in blue. Cosmogenic activation products are colored red. Green denotes backgrounds from the environment or those introduced during detector assembly. Purple is for $\mu$-induced backgrounds at depth. An upper limit on the negligible background from atmospheric and other neutrinos is shown in orange. The contributions sum to $3.0 \mathrm{cnts} /(\mathrm{ROI}-\mathrm{t}-\mathrm{y})$ in the Majorana Demonstrator.

5.4. Predicted Sensitivity of the Majorana Demonstrator. The sensitivity of a neutrinoless double-beta decay search increases with the exposure of the experiment but ultimately depends on the achieved background level. This relationship is illustrated for the Demonstrator in Figure 14, in which we have used the Feldman-Cousins [70] definition of sensitivity in order to transition smoothly between the backgroundfree and background-dominated regimes. The background expectation for the Demonstrator is $3 \mathrm{cnts} /(\mathrm{ROI}-\mathrm{t}-\mathrm{y})$ in a $4 \mathrm{keV}$ energy window at the $\beta \beta(0 \nu)$-decay endpoint energy. A minimum exposure of about $30 \mathrm{~kg}-\mathrm{y}$ is required to test the recent claim of an observation of $\beta \beta(0 \nu)$ decay [19].

\section{Status, Prospects, and Conclusions}

6.1. Status of the Majorana Demonstrator Project. The MAJORANA collaboration obtained beneficial occupancy of its Davis Campus underground laboratories in May 2012. At the time of this writing in June 2013, the collaboration has completed outfitting the labs, established cleanliness (the detector room is typically better than 500 particles $/ \mathrm{ft}^{3}$ of diameter $0.5 \mu \mathrm{m}$ or smaller), and is proceeding with the construction and assembly of the array. The underground electroforming laboratories, which started operation in the summer of 2011, have now produced more than $75 \%$ of the required copper. The $42.5 \mathrm{~kg}$ of $86 \%$ enriched ${ }^{76} \mathrm{Ge}$ has been reduced from $\mathrm{GeO}_{2}$ and refined to electronic grade $\mathrm{Ge}$ with a yield of $98 \%$. Ten enriched P-PC detectors with a total mass of $9.5 \mathrm{~kg}$ have been produced by ORTEC, with eight now underground to SURF. A prototype cryostat, the same as the ultraclean cryostats, but fabricated from commercial copper, has been assembled and operated with its associated vacuum system. Two strings of natural Ge detectors have been built in the glove boxes and are undergoing testing before they are installed in the prototype cryostat. Cryostat 1 has been machined from the UGEFCu. Samples obtained from all materials being used in the DEMONSTRATOR are being assayed. Slow-control systems and their associated sensors are in continuous operation in all UG laboratories. Data acquisition for detector acceptance testing, string testing, and the main array are operational.

The prototype cryostat will be commissioned in the summer of 2013. Cryostat 1, which will contain seven strings of both enriched and natural Ge detectors, is scheduled to be completed in late 2013. Cryostat 2, which is expected to contain all enriched detectors, is expected to be assembled in 2014. The full array should be in operation in 2015. The DEMONSTRATOR will be operated for about 3 years in order to collect $\sim 100 \mathrm{~kg}$-years of exposure.

6.2. The Future Large-Scale Experiment. The Majorana and GERDA collaborations are working together to prepare for a single-tonne-scale ${ }^{76} \mathrm{Ge}$ experiment that will combine the best technical features of both experiments. The results of the two experiments will be used to determine the best path forward.

The present generation of experiments will likely produce results with limits on $\left\langle m_{\beta \beta}\right\rangle$ below about $100 \mathrm{meV}$. The next generation will strive to cover the inverted hierarchy region of the effective Majorana neutrino mass. To accomplish this, 


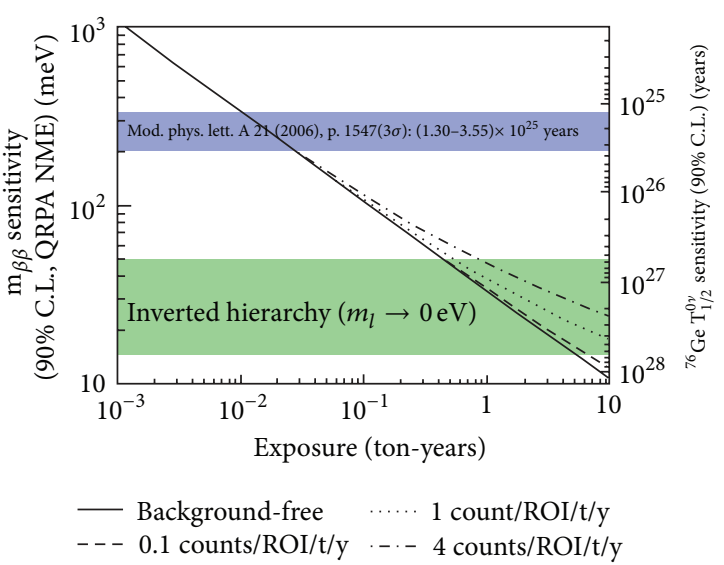

FIGURE 14: $90 \%$ C.L. sensitivity as a function of exposure for $\beta \beta(0 \nu)$ decay searches in ${ }^{76} \mathrm{Ge}$ under different background scenarios. Matrix elements from [71] were used to convert half-life to neutrino mass. The blue band shows the region where a signal would be detected if the recent claim [19] is correct.

an experiment will require $\left\langle m_{\beta \beta}\right\rangle$ sensitivity down to about $20 \mathrm{meV}$. Such small neutrino masses would indicate a halflife longer than $10^{27}$ y. As seen in Figure 14, to observe such a long half-life, one will need a tonne or more of isotope and backgrounds below $1 \mathrm{cnt} /(\mathrm{ROI}-\mathrm{t}-\mathrm{y})$.

\section{Conflict of Interests}

The authors declare that there is no conflict of interests regarding the publication of this paper.

\section{Acknowledgments}

The authors acknowledge support from the Office of Nuclear Physics in the DOE Office of Science under Grant nos. DE-AC02-05CH11231, DE-FG02-97ER41041, DE-FG0297ER41033， DE-FG02-97ER4104， DE-FG02-97ER41042, DE-SCOO05054, DE-FG02-10ER41715, and DE-FG0297ER41020. They also acknowledge support from the Particle and Nuclear Astrophysics Program of the National Science Foundation through Grant nos. PHY-0919270, PHY1003940, 0855314, PHY-1202950, MRI 0923142, and 1003399. They gratefully acknowledge support from the Russian Foundation for Basic Research Grant no. 12-02-12112 and the support of the U.S. Department of Energy through the LANL/LDRD Program.

\section{References}

[1] Y. Ashie, J. Hosaka, and K. Ishihara, "Evidence for an oscillatory signature in atmospheric neutrino oscillations," Physical Review Letters, vol. 93, no. 10, Article ID 101801, 6 pages, 2004.

[2] S. N. Ahmed, A. E. Anthony, and E. W. Beier, "Measurement of the total active ${ }^{8} \mathrm{~B}$ solar neutrino flux at the Sudbury Neutrino Observatory with enhanced neutral current sensitivity," Physical Review Letters, vol. 92, no. 18, Article ID 181301, 6 pages, 2004.
[3] S. Abe, T. Ebihara, and S. Enomoto, "Precision measurement of neutrino oscillation parameters with KamLAND," Physical Review Letters, vol. 100, no. 22, Article ID 221803, 5 pages, 2008.

[4] E. Majorana, "Teoria simmetrica dell'elettrone e del positrone," Nuovo Cimento, vol. 5, no. 4, pp. 171-184, 1937, English Translation in Soryushiron Kenkyu, vol. 63, p. 149, 1981.

[5] T. Yanagida, "Horizontal gauge symmetry and masses of neutrinos," in Proceedings of the Workshop on Unified Theory and Baryon Number in the Universe, O. Sawada and A. Sugamoto, Eds., KEK, Tsukuba, Japan, 1979.

[6] M. Gell-Mann, P. Ramond, and R. Slansky, Supergravity, NorthHolland, Amsterdam, The Netherlands, 1979.

[7] M. Fukugita and T. Yanagida, "Barygenesis without grand unification,” Physics Letters B, vol. 174, no. 1, pp. 45-47, 1986.

[8] P. Di Bari, "An introduction to leptogenesis and neutrino properties," Contemporary Physics, vol. 53, no. 4, pp. 315-338, 2012.

[9] M. Zralek, "On the possibilities of distinguishing dirac from Majorana neutrinos," Acta Physica Polonica B, vol. 28, no. 11, pp. 2225-2257, 1997.

[10] J. Schechter and J. W. F. Valle, "Neutrinoless double- $\beta$ decay in $\mathrm{SU}(2) \times \mathrm{U}(1)$ theories," Physical Review D, vol. 25, no. 11, pp. 2951-2954, 1982.

[11] L. Baudis, A. Dietz, G. Heusser et al., "Limits on the Majorana neutrino mass in the $0.1 \mathrm{eV}$ range," Physical Review Letters, vol. 83 , no. 1, pp. 41-44, 1999.

[12] C. E. Aalseth, F. T. Avignone III, R. L. Brodzinski et al., "Neutrinoless double- $\beta$ decay of ${ }^{76} \mathrm{Ge}$ : first results from the International Germanium Experiment (IGEX) with six isotopically enriched detectors," Physical Review C, vol. 59, no. 4, pp. 21082113, 1999.

[13] C. E. Aalseth, F. T. Avignone III, R. L. Brodzinski et al., "IGEX ${ }^{76} \mathrm{Ge}$ neutrinoless double-beta decay experiment: prospects for next generation experiments," Physical Review D, vol. 65, no. 9, Article ID 092007, 6 pages, 2002.

[14] C. E. Aalseth, F. T. Avignone III, R. L. Brodzinski et al., "The IGEX experiment reexamined: a response to the critique of Klapdor-Kleingrothaus, Dietz, and Krivosheina," Physical Review D, vol. 70, no. 7, Article ID 078302, 5 pages, 2004.

[15] N. Ackerman, B. Aharmim, M. Auger et al., "Observation of two-neutrino double-beta decay in ${ }^{136} \mathrm{Xe}$ with the EXO-200 detector," Physical Review Letters, vol. 107, no. 21, Article ID 212501, 5 pages, 2011.

[16] M. Auger, D. J. Auty, P. S. Barbeau et al., "Search for neutrinoless double-beta decay in ${ }^{136} \mathrm{Xe}$ with EXO-200," Physical Review Letters, vol. 109, no. 3, Article ID 032505, 6 pages, 2012.

[17] A. Gando, Y. Gando, H. Hanakago et al., "Measurement of the double- $\beta$ decay half-life of ${ }^{136} \mathrm{Xe}$ with the KamLAND-Zen experiment," Physical Review C, vol. 85, no. 4, Article ID 045504, 6 pages, 2012.

[18] A. Gando, Y. Gando, H. Hanakago et al., "Limit on neutrinoless $\beta \beta$ decay of ${ }^{136} \mathrm{Xe}$ from the first phase of KamLAND-Zen and comparison with the positive claim in ${ }^{76} \mathrm{Ge}, "$ Physical Review Letters, vol. 110, no. 6, Article ID 062502, 5 pages, 2013.

[19] H. V. Klapdor-Kleingrothaus and I. V. Krivosheina, "The evidence for the observation of $0 \nu \beta \beta$ decay: the identification of $0 v \beta \beta$ events from the full spectra," Modern Physics Letters A, vol. 21, no. 20, pp. 1547-1566, 2006.

[20] C. E. Aalseth, F. T. Avignone III, A. Barabash et al., "Comment on "evidence for neutrinoless double beta decay'”, Modern Physics Letters A, vol. 17, no. 22, p. 1475, 2002. 
[21] Y. G. Zdesenko, F. A. Danevich, and V. I. Tretyak, "Has neutrinoless double $\beta$ decay of ${ }^{76} \mathrm{Ge}$ been really observed?" Physics Letters B, vol. 546, no. 3-4, pp. 206-215, 2002.

[22] F. Feruglio, A. Strumia, and F. Vissani, "Neutrino oscillations and signals in $\beta$ and $0 \nu 2 \beta$ experiments," Nuclear Physics B, vol. 637, no. 1-3, pp. 345-377, 2002.

[23] M. Agostini, M. Allardt, E. Andreotti et al., "Results on neutrinoless double- $\beta$ decay of ${ }^{76} \mathrm{Ge}$ from phase I of the GERDA experiment," Physical Review Letters, vol. 111, no. 12, Article ID 122503, 6 pages, 2013.

[24] M. Agostini, M. Allardt, E. Andreotti et al., "The background in the neutrinoless double beta decay experiment GERDA," http:// arxiv.org/abs/1306.5084.

[25] M. Agostini, M. Allardt, E. Andreotti et al., "Pulse shape discrimination for GERDA phase I data," The European Physical Journal C, vol. 73, article 2583, 2013.

[26] S. R. Elliott and P. Vogel, "Double beta decay," Annual Review of Nuclear and Particle Science, vol. 52, pp. 115-151, 2002.

[27] S. R. Elliott and J. Engel, "Double-beta decay," Journal of Physics G, vol. 30, no. 9, article 183, 2004.

[28] A.S. Barabash, "Double-beta-decay experiments: present status and prospects for the future," Physics of Atomic Nuclei, vol. 67, no. 3, pp. 438-452, 2004.

[29] H. Ejiri, "Double beta decays and neutrino masses," Journal of the Physical Society of Japan, vol. 74, pp. 2101-2127, 2005.

[30] F. T. Avignone III, G. S. King III, and Y. G. Zdesenko, "Next generation double-beta decay experiments: metrics for their evaluation," New Journal of Physics, vol. 7, article 6, no. 1, 2005.

[31] F. T. Avignone III, S. R. Elliott, and J. Engel, "Double beta decay, Majorana neutrinos, and neutrino mass," Reviews of Modern Physics, vol. 80, no. 2, pp. 481-516, 2008.

[32] A. S. Barabash, "Double beta decay experiments," Physics of Particles and Nuclei, vol. 42, no. 4, pp. 613-627, 2011.

[33] W. Rodejohann, "Neutrino-less double beta decay and particle physics," International Journal of Modern Physics E, vol. 20, no. 9, article 1833, 2011.

[34] S. R. Elliott, "Recent progress in double beta decay," Modern Physics Letters A, vol. 27, no. 7, Article ID 1230009, 16 pages, 2012.

[35] J. D. Vergados, H. Ejiri, and F. Šimkovic, "Theory of neutrinoless double-beta decay," Reports on Progress in Physics, vol. 75, no. 10, Article ID 106301, 2012.

[36] J. Beringer, J.-F. Arguin, R. M. Barnett et al., "Review of particle physics," Physical Review D, vol. 86, no. 1, Article ID 010001, 1528 pages, 2012.

[37] K.-H. Ackermann, M. Agostini, M. Allardt et al., "The GERDA experiment for the search of $0 \nu \beta \beta$ decay in ${ }^{76} \mathrm{Ge}$," The European Physical Journal C, vol. 73, article 2330, 2013.

[38] C. E. Aalseth, P. S. Barbeau, D. G. Cerdeño et al., "Experimental constraints on a dark matter origin for the dama annual modulation effect," Physical Review Letters, vol. 101, no. 25, Article ID 251301, 4 pages, 2008.

[39] C. E. Aalseth, P. S. Barbeau, N. S. Bowden et al., "Results from a search for light-mass dark matter with a $p$-type point contact germanium detector," Physical Review Letters, vol. 106, no. 13, Article ID 131301, 4 pages, 2011.

[40] C. E. Aalseth, P. S. Barbeau, J. Colaresi et al., "Search for an annual modulation in a $p$-type point contact germanium dark matter detector," Physical Review Letters, vol. 107, no. 14, Article ID 141301, 5 pages, 2011.
[41] C.E. Aalseth, M. Amman, F. T. Avignone III et al., "Astroparticle physics with a customized low-background broad energy germanium detector," Nuclear Instruments and Methods in Physics Research A, vol. 652, no. 1, pp. 692-695, 2011.

[42] R. Agnese, Z. Ahmed, A.J. Anderson et al., "Silicon detector dark matter results from the final exposure of CDMS II," Physical Review Letters, vol. 111, no. 25, Article ID 251301, 6 pages, 2013.

[43] G. K. Giovanetti, E. Aguayo, F. T. Avignone et al., "Dark matter sensitivities of the Majorana Demonstrator," Journal of Physics, vol. 375, no. 1, Article ID 012014, 2012.

[44] F. T. Avignone III, D. Abriola, R. L. Brodzinski et al., "Experimental search for solar axions via coherent Primakoff conversion in a germanium spectrometer," Physical Review Letters, vol. 81, no. 23, pp. 5068-5071, 1998.

[45] R. J. Creswick, F. T. Avignone III, H. A. Farach et al., "Theory for the direct detection of solar axions by coherent Primakoff conversion in germanium detectors," Physics Letters B, vol. 427, no. 3-4, pp. 235-240, 1998.

[46] A. V. Derbin, A. S. Kayunov, V. V. Muratova et al., "Constraints on the axion-electron coupling for solar axions produced by a Compton process and bremsstrahlung," Physical Review D, vol. 83, no. 2, Article ID 023505, 7 pages, 2011.

[47] F. T. Avignone III, "Potential for large germanium detector arrays for solar-axion searches utilizing the axio-electric effect for detection," Physical Review D, vol. 79, no. 3, Article ID 035015, 5 pages, 2009.

[48] S. R. Elliott, B. H. LaRoque, V. M. Gehman, M. F. Kidd, and M. Chen, "An improved limit on Pauli-exclusion-principle forbidden atomic transitions," Foundations of Physics, vol. 42, no. 8, pp. 1015-1030, 2012.

[49] P. S. Barbeau, J. I. Collar, and O. Tench, "Large-mass ultralow noise germanium detectors: performance and applications in neutrino and astroparticle physics," Journal of Cosmology and Astroparticle Physics, vol. 2007, no. 9, article 009, 2007.

[50] K. Scholberg, "Prospects for measuring coherent neutrinonucleus elastic scattering at a stopped-pion neutrino source," Physical Review D, vol. 73, no. 3, Article ID 033005, 9 pages, 2006.

[51] A. J. Anderson, J. M. Conrad, E. Figueroa-Feliciano, K. Scholberg, and J. Spitz, "Coherent neutrino scattering in dark matter detectors," Physical Review D, vol. 84, no. 1, Article ID 013008, 2011.

[52] P. N. Luke, F. S. Goulding, N. W. Madden, and R. H. Pehl, "Low capacitance large volume shaped-field germanium detector," IEEE Transactions on Nuclear Science, vol. 36, no. 1, pp. 926930, 1989.

[53] "Broad energy Ge detectors," Canberra Industries, Meriden, Conn, USA, 2009.

[54] “ORTEC”, Oak Ridge, Tenn, USA, 2009.

[55] D. Budjáš, M. B. Heider, O. Chkvorets, N. Khanbekov, and S. Schönert, "Pulse shape discrimination studies with a broadenergy germanium detector for signal identification and background suppression in the GERDA double beta decay experiment," Journal of Instrumentation, vol. 4, no. 10, Article ID P10007, 2009.

[56] R. Cooper, D.C. Radford, K. Lagergren et al., "A Pulse shape analysis technique for the Majorana experiment," Nuclear Instruments and Methods in Physics Research A, vol. 629, no. 1, pp. 303-310, 2011. 
[57] E. Aguayo, M. Amman, F.T. Avignone III et al., "Characteristics of signals originating near the lithium-diffused $\mathrm{N}+$ contact of high purity germanium p-type point contact detectors," Nuclear Instruments and Methods in Physics Research A, vol. 701, pp. 176185, 2013.

[58] P. Finnerty, A direct dark matter search with the Majorana lowbackground broad energy germanium detector [Ph.D. thesis], University of North Carolina, Chapel Hill, NC, USA, 2013.

[59] E. Aguayo, J. E. Fast, R. T. Kouzes, and J. L. Orrell, “The /spl mu/ -witness detector: a ruggedized, portable, flux meter for cosmogenic activation monitoring," IEEE Transactions on Nuclear Science, vol. 60, no. 2, pp. 689-692, 2013.

[60] E. W. Hoppe, E. E. Mintzer, C. E. Aalseth et al., "Microscopic evaluation of contaminants in ultra-high purity copper," Journal of Radioanalytical and Nuclear Chemistry, vol. 282, no. 1, pp. 315-320, 2009.

[61] N. R. Overman, D. J. Edwards, C. T. Overman, E. W. Hoppe, and T. A. Kafentzis, "Majorana electroformed copper mechanical analysis," Tech. Rep. PNNL-21315, Pacific Northwest National Laboratory, 2012.

[62] E. Aguayo, M. Busch, R. Daniels, J. E. Fast, M. P. Green, and D. J. Reid, "The design of an ultra-low background thermosyphon for the Majorana Demonstrator," Nuclear Instruments and Methods in Physics Research A, vol. 709, pp. 17-21, 2013.

[63] P. Barton, P. Luke, M. Amman et al., "Low-noise low-mass front end electronics for low-background physics experiments using germanium detectors," in Proceedings of the IEEE Nuclear Science and Medical Imaging Conference (NSS/MIC '11), pp. 1976-1979, Valencia, Spain, 2011.

[64] S. Zimmermann, J.T. Anderson, D. Doering et al., "Implementation and performance of the electronics and computing system of the gamma ray energy tracking in-beam nuclear array (GRETINA)," IEEE Transactions on Nuclear Science, vol. 59, no. 5, pp. 2494-2500, 2012.

[65] M. A. Howe, G. A. Cox, P. J. Harvey et al., "Sudbury neutrino observatory neutral current detector acquisition software overview," IEEE Transactions on Nuclear Science, vol. 51, no. 3, pp. 878-883, 2004.

[66] J. Heise, "Private communication," 2013.

[67] S. Agostinelli, J. Allison, K. Amako et al., "Geant4-a simulation toolkit," Nuclear Instruments and Methods in Physics Research A, vol. 506, no. 3, pp. 250-303, 2003.

[68] J. Allison, K. Amako, J. Apostolakis et al., "GeAnt4 developments and applications," IEEE Transactions on Nuclear Science, vol. 53, no. 1, pp. 270-278, 2006.

[69] M. Boswell, Y.-D. Chan, J. A. Detwiler et al., "MaGe-a Geant4based Monte Carlo application framework for low-background germanium experiments," IEEE Transactions on Nuclear Science, vol. 58, no. 3, pp. 1212-1220, 2011.

[70] G. J. Feldman and R. D. Cousins, "Unified approach to the classical statistical analysis of small signals," Physical Review D, vol. 57, no. 7, pp. 3873-3889, 1998.

[71] F. Šimkovic, A. Faessler, H. Müther, V. Rodin, and M. Stauf, " $0 \nu \beta \beta$-decay nuclear matrix elements with self-consistent short-range correlations," Physical Review C, vol. 79, no. 5, Article ID 055501, 10 pages, 2009. 

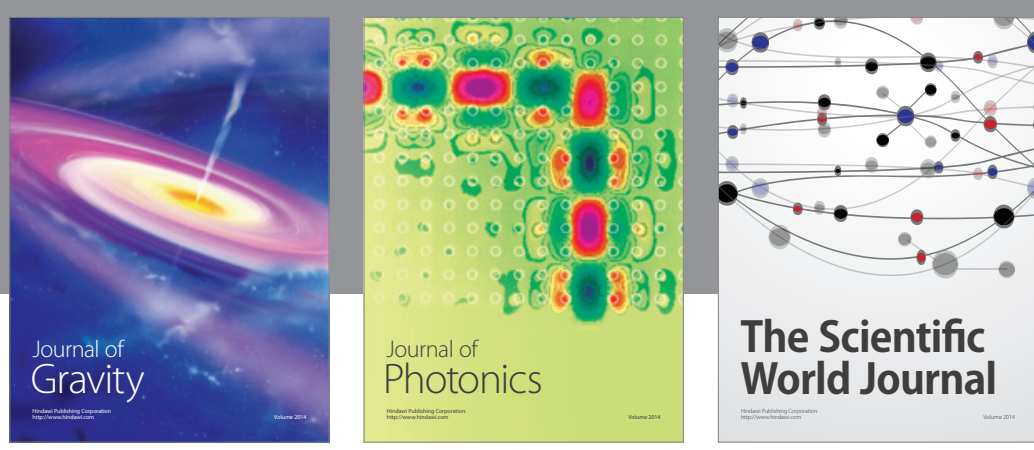

The Scientific World Journal
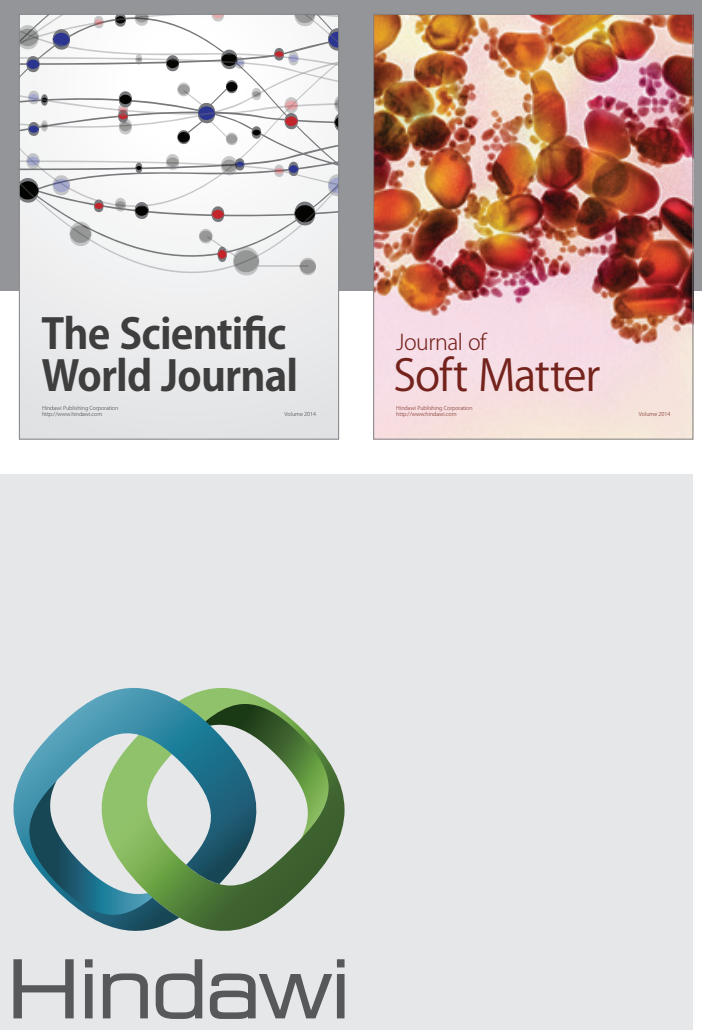

Submit your manuscripts at

http://www.hindawi.com

nternational Journal of

Statistical Mechanics
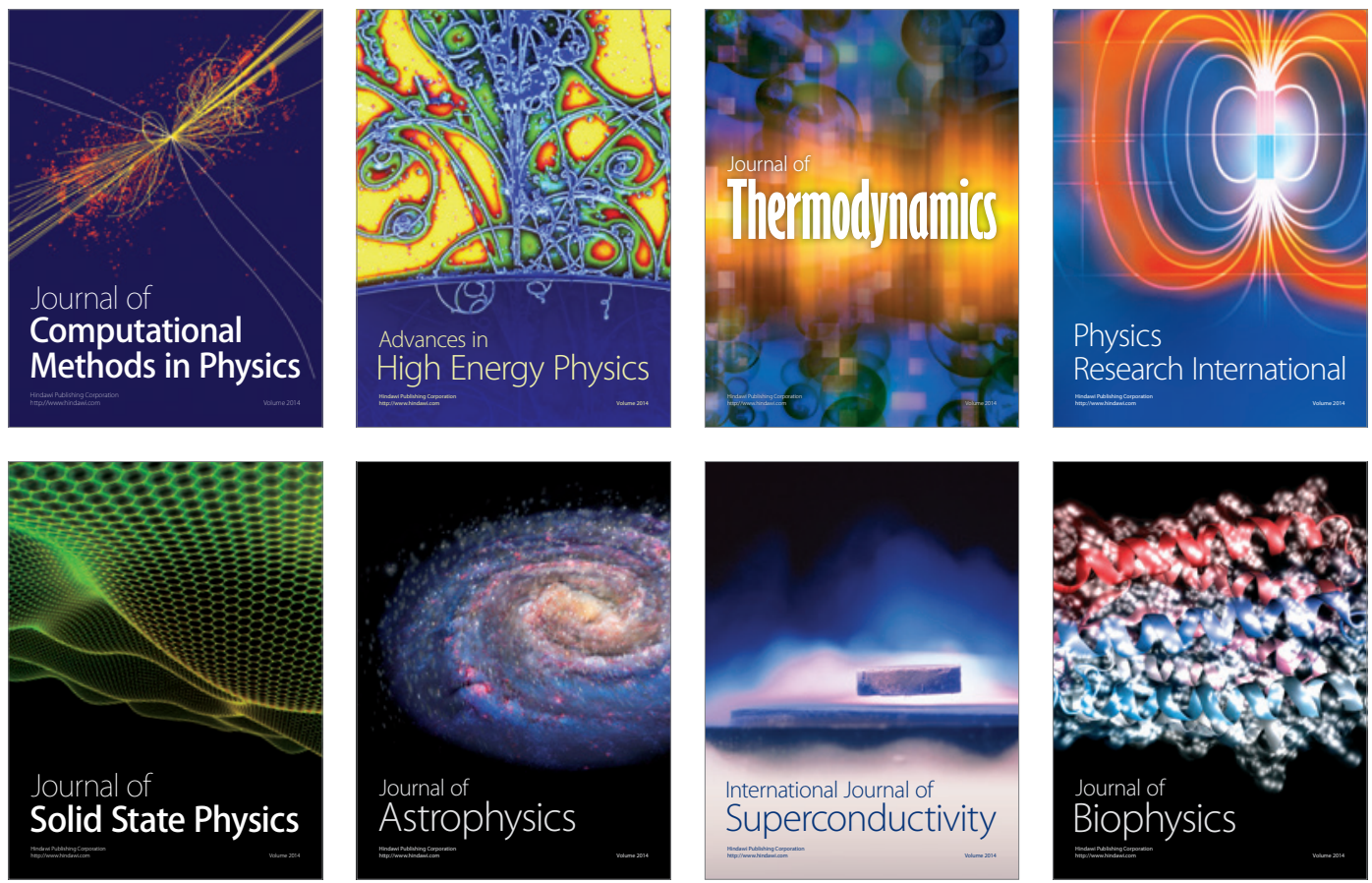
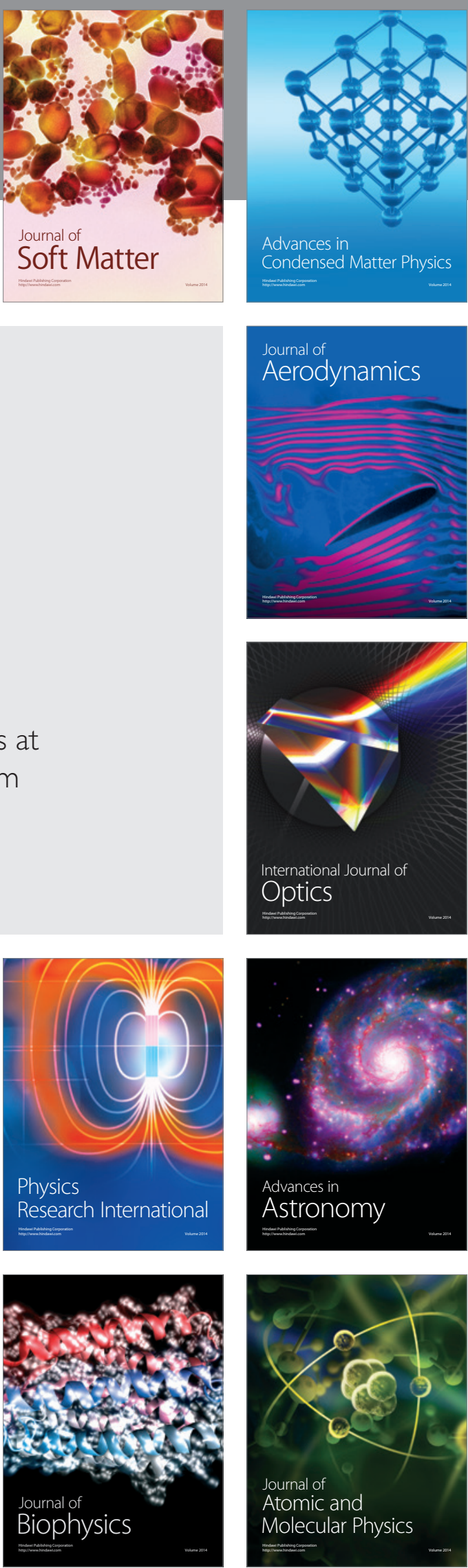